\title{
New genomic signals underlying the emergence of human proto- genes
}

\author{
Anna Grandchamp ${ }^{1 *}$, Katrin Berk ${ }^{1}$, Elias Dohmen ${ }^{1}$ and Erich Bornberg-Bauer ${ }^{1}$ \\ $1 \quad$ Affiliation 1; Institute for Evolution and Biodiversity, Westfälische Wilhelms University, Hüfferstrasse 1, 48149 \\ Münster, Germany \\ * Correspondence: a.grandchamp@uni-muenster.de
}

\begin{abstract}
De novo genes are novel genes which emerge from non-coding DNA. Until now, little is known about de novo genes properties, correlated to their age and mechanisms of emergence. In this study, we investigate four properties: introns, upstream regulatory motifs, 5' UTRs and protein domains, in 23135 human proto-genes. We found that proto-genes contain introns, whose number and position correlates with the genomic position of proto-gene emergence. The origin of these introns is debated, as our result suggest that $41 \%$ proto-genes might have captured existing introns, as well as the fact that $13.7 \%$ of them do not splice the ORF. We show that proto-genes which emerged via overprinting tend to be more enriched in core promotor motifs, while intergenic and intronic ones are more enriched in enhancers, even if the motif TATA is most expressed upstream these genes. Intergenic and intronic 5' UTRs of protogenes have a lower potential to stabilise mRNA structures than exonic proto-genes and established human genes. Finally, we confirm that proto-genes gain new putative domains with age. Overall, we find that regulatory motifs inducing transcription and translation of previously non-coding sequences may facilitate proto-gene emergence. Our paper demonstrates that introns, 5'UTRs, and domains have specific properties in proto-genes. We also show the importance of studying proto-genes in relation to their genomic position, as it strongly impacts these properties.
\end{abstract}

Keywords: human proto-genes, introns, regulatory motifs, 5’UTRs, protein domains

\section{Introduction}

De novo gene birth is defined as the emergence of a new gene from previously non-coding sequences [1,2,3,4]. Over the past 15 years, an increasing amount of studies investigated de novo gene birth and its role in genome evolution [3,5,6,7]. The emergence of new genes depends mainly on two processes: The occurrence of a transcription event in a previously untranscribed DNA region, and the emergence of an open reading frame (ORF) encoding for a protein [6]. Putative de novo genes which appeared via the emergence of an ORF and a transcription event is called a protogene [8]. The mechanisms of proto-gene emergence differ depending on the genomic location in which the new gene emerges.

Among these mechanisms, overprinting is characterised by the overlap of a new gene with preexisting exons $[9,10]$. If the overlap is in frame with the pre-existing exon, the resulting protein will be shorter or longer than the original one. If it is not in frame, or on the complementary DNA strand, a completely new protein can emerge [11,12]. Overprinting can lead to other events. For example, de novo extension of an exonic open reading frame (ORF) can give rise to the overlap of distant genes. This process affects 5 to $14 \%$ of the vertebrate genes [13]. 
Exonisation characterises the emergence of a new gene which overlaps with an intron $[14,15]$. The minimum requirement for such an event to occur is the retention of an intron as an exonic sequence, and the presence of an ORF inside. Proto-genes can also emerge "from scratch" in intergenic regions. This occurs when a transcript emerges from a previously untranscribed intergenic region, which also gains an ORF [16,17]. Emergences "from scratch" are difficult to explain, as all constitutive elements of a gene need to be present simultaneously. Since all sequence signals which are not constantly under purifying selection are likely to be lost again, their co-occurrence may be a consequence of their emergence in close temporal proximity. Accordingly, such an emergence must be considered very unlikely and certainly deserves more detailed investigations. Two additional mechanisms have been proposed to account for proto-gene emergence: An ORF can emerge in a non-coding RNA (ncRNA), or a pseudogene can regain transcription [2]. However, we will focus on the three first mechanisms in this manuscript: overprinting, exonisation and emergence "from scratch".

So far, several de novo genes have been shown to provide important functionality, as for example playing a role in reproduction $[18,19]$, adaptation to new ecological niches (ex antifreeze proteins enabling fish to survive in arctic water [20], speciation events, etc [21,22,23]. A major limitation in studying de novo genes is the difficulty to ascertain that a proto-gene arose de novo, and did not emerge through rapid evolution or transposition of a previously existing gene. To avoid false positives in de novo gene identification, which is mainly due to divergence of homologues, detection procedures have been constantly refined [24,25]. In essence, to accomplish a high degree of selectivity, rigorous methods such as synteny and similarity profile searches need to be applied $[5,26,27]$. De novo proteins have been shown to contain a high level of intrinsic structural disorder and lack a stable three-dimensional structure [28], even if that result has been challenged by [19], who did not observed distinguishable properties between de novo gene structure and randomly selected sequences. By studying viruses, [10] demonstrated that de novo genes which appeared via overprinting may have a different codon usage than established gene. Some de novo genes preferentially emerge next to promotors in GC-rich intergenic regions and can make use of bidirectional promotors [29]. De novo genes in plants have been shown to be more prone to emerge when they have a higher epigenetic methylation state [30]. De novo genes have also been shown to be enriched in transcription factors [23]. The expression of young genes is lower than of established genes, and more tissue-specific [7,8]. Based on an evolutionary perspective, the older de novo genes become, the more pervasively expressed they are [31], and the more selected [32]. However, the above-mentioned properties are not sufficient to distinguish proto-genes from new genes which have emerged through other mechanisms. Investigating new features of proto-genes may allow to make such a distinction, and improve the basic knowledge of de novo genes. 
In this study, we aim to characterise properties of proto-genes, which have to the best of our knowledge not been reported before, in order to improve their detection and description. Secondly, we aim to understand if the three mechanisms of emergence ("overprinting”, "exonisation”, or “from scratch”), as well as the approximate age of emergence, influence the properties of the protogenes. We investigated four properties by studying 27550 proto-genes from the human lineage published by [33] : (1) the presence of introns, (2) the enrichment and nature of motifs specific for transcription, (3) the properties of 5' untranslated region (UTR), and (4) the presence of protein domains.

We first studied the presence of introns (1). Introns are major constituents in eukaryote genomes, as they allow for alternative splicing, and therefore considerably increase the number of encoded proteins compared to the total number of genes [34]. Introns can be spliced by RNA catalyses, by proteins, or by the spliceosome [35,36,37]. In extant eukaryotes, the emergence of introns in genes can be described by different scenarios [38]: Intron transposition, transposon insertion, tandem duplication, intron gain during double strand break repair, insertion of a group2 intron, intron transfer, and intronisation. De novo genes are mostly thought to not contain introns $[5,39]$ However, this assumption is based on small datasets. In this present manuscript, we examine if proto-genes contain introns, and if intron size and number is correlated to the age and the mechanism of emergence of the proto-gene.

Secondly, we investigate the enrichment and nature of DNA motifs specific for transcription of proto-genes (2). The mechanism of gene transcription is well known and includes complex binding of transcription factors in eukaryotes [40]. The binding of transcription factors is facilitated by specific DNA motifs in the promotor and in enhancer regions. The overall transcription is known to be less pervasive in proto-genes compared to older established genes [3]. However, little is known about the presence of motifs allowing the recruitment of the transcription machinery.

Third, we studied 5' UTRs (3) of proto-genes, as they are essential elements of the translation machinery of a gene [41,42]. In eukaryotes, the 5' UTR, which is located upstream of the start codon, contains the Kozac sequence [43] which is recognised by the ribosome supported by multiple factors. Furthermore, UTRs play a role in a couple of biological processes, including modulating of mRNA transport out of the nucleus, or subcellular localisation [44]. 5' UTRs are GCrich in eukaryotes, which supports stable secondary structure elements playing a role in the transcription regulation [45,46]. Moreover, it has been proven that mutations in UTRs can deregulate transcription. Additionally, some diseases are caused by mutation in UTRs [47]. In the present manuscript, we investigate the folding potential of the 5' UTR in proto-genes and compare it to the folding potential of established human genes. 
In a last step, we studied protein domains (4). Domains are structural or functional units of proteins which fold in an independent way $[48,49]$. Most domains are shared by many proteins and organisms [50], and rearrangements of their order are frequent [51,52]. De novo genes are thought to not contain any known domains [25]. However, as domains rearrange through different molecular mechanisms, de novo genes might acquire existing domains, e.g. by fusion with another, long existing gene coding for such a domain. In the present study, we investigate if proto-genes contain known domains, and if there is a correlation between the presence of a known domain in protogenes and their age and mechanism of emergence. We also investigate if putative proto- proteins contain novel domains. This would be conceivable, considering that a recent study found that, presumably via occasional read through [53] it is possible that a reading frame becomes extended [54] and eventually some of the extended fragments become stabilised by forming hydrophic interactions [55].

We used the four properties (presence of introns, enrichment and nature of motifs specific for transcription, properties of 5' UTR, and presence of domains,) to compare proto-genes emerged de novo to established human genes, in order to determine if the mentioned properties allow a distinction between the two gene classes. Moreover, we classified proto-genes according to their genomic position which depict their mechanism of emergence (“overprinting”, "exonisation”, or “from scratch"), as well as by their evolutionary age.

\section{Method}

\subsection{Datasets}

The human genome GRCh38 and its corresponding GTF file were downloaded from Ensembl [56]. The 27491 putative human de novo transcribed ORFs, nucleotide sequences and corresponding protein sequences, were provided by [33]. ORFs with an unusual start or stop codon are only included in the domain annotation, but were removed for the other analyses, giving rise to a file of 23135 putative human de novo transcribed ORFs. The spliced transcripts containing de novo transcribed ORFs as well as the genomic positions and exon-intron structure of the corresponding unspliced transcripts (GTF file) were provided by [33]. Each de novo transcript containing a de novo ORF is here described as a proto-gene. Proteins encoded by putative proto-genes are mentioned as proto-proteins. The genomic contexts in which the proto-genes emerged are described in this study as "genomic position". The three genomic positions directly refer to the mechanism of ORF emergence: “exonic” ORFs = overlap with an annotated exon (overprinting). "intronic” ORFs = overlap with an intron (exonisation), “intergenic” ORFs = emerged inside a non-coding region and therefore from scratch. Ages of the proto-genes were annotated as following: I0 = specific to 
humans (Homo sapiens); I1 = emergence at 6.65 Mya, shared between humans, bonobo (Pan paniscus), and chimpanzee (Pan troglodytes); I2 = 9.06 Mya (I1+gorilla (Gorilla gorilla)); I3 = 15.76 Mya (I2+orang-utan (Pongo pygmaeus)); I4 = 29.44 Mya (I3+rhesus macaque (Macaca mulatta)); I5 = 90 Mya (I4+mouse (Mus musculus) as outgroup)). Proto-genes from age class I5 are not primate-restricted, but they are considered as outgroup proto-genes in the present study.

\subsection{Intron search}

The exon/intron structure of the unspliced transcript of all proto-genes was extracted from the GTF file of the transcriptome assembly. The corresponding intronic sequences were retrieved from the human reference genome. The average number of introns per transcript was determined for each proto-gene, as well as their size, and were compared according to the age and the genomic position of the proto-gene, respectively.

From proto-genes containing at least one intron, we retrieved the ones from which the ORFs were located on one single exon and therefore not affected by splicing, and compared them according to their age and their genomic position.

Since we investigated if introns from “intergenic" proto-genes may result from the recycling of already existing human introns, we extracted all introns present in proto-genes from the genomic location "intergenic", and randomly selected a subset of 500. The coordinates of the human introns from established genes were determined by using the UCSC table browser [57], and the FASTA sequences of all annotated introns were retrieved accordingly.

The 500 randomly selected introns from "intergenic" proto-genes were used as query for a BLASTn search against all annotated human introns. We extracted all BLAST hits with an E-value $<=0.01$, a percentage of ID > to $80 \%$, and >= $80 \%$ length overlap between query and database sequence.

\subsection{Motif search}

We investigated the presence of transcription factor motifs upstream of the transcription starting point of proto-genes. Transcription can be regulated at different levels: by promotors and by enhancer sequences [21]. We searched for transcription factor motifs by using a database from JASPAR [58], which contains 746 annotated vertebrate specific transcription factor binding motifs from promotors and enhancers. For simplicity, we refer to this database as “MotifsAllUpstream”. We then scanned DNA regions located 200 base pairs (bp) upstream of the transcription starting site (TSS) of proto-genes for the previously detected motifs. $200 \mathrm{bp}$ upstream of the TSS cover the promotor and part of proximal regulation. The transcription starting points were accessed through the gtf file of the transcriptome assembly. The 200 bp sequences upstream of the TSS were downloaded for each of the 23135 proto-genes without an unusual start codon (subset 1). 
Additionally, we retrieved the same regions for all established human coding genes (Ensembl = 21445, subset 2), pseudogenes (Ensembl = 15200, subset 3), and non-coding RNA (ncRNA, Ensembl $=23934$, subset 4$)$. The transcription starting point of the genes were retrieved from the GTF file of the human genome.

As a control for comparison, we searched these motifs in two more datasets: one dataset with non coding regions and another one with intronic sequence. To build these datasets, we first calculated the positions of all non-coding regions and intronic regions in the human genome by using the GTF file and retrieving the corresponding FASTA sequences. Secondly, we randomly picked 23135 regions (as much as proto-genes) of 200 bp from all non-coding regions (subset 5) and intronic regions (subset 6). Overlaps between the selected regions were excluded, as well as overlaps with “intronic" and "intergenic" proto-genes. We compared the number of motifs found in the six subsets of upstream sequences.

As a second analyses, we also search for motifs specifically for core promotors, by using another JASPAR database containing nine motifs from core promotors. We refer to this database as “MotifsCore”. Such motifs are located between about 50 nucleotides before and after the TSS [59]. We therefore analysed DNA sequences from 100 bp before to $100 \mathrm{bp}$ after the TSS of each protogene. We additionally looked at core motif regions (100 bp up and downstream of TSS) from established human coding genes, pseudogenes, and ncRNAs. We also searched for these regions in the previously randomly selected intronic and intergenic 200 bp sequences.

By comparing the two JASPAR motifs databases with the respective six subsets of DNA sequences using the biopython package [60], we searched for the presence of motifs within the latter. For each detected motif, a position-weight was calculated, followed by a position-specific scoring matrix (PSSM). We used PSSM score to search for the motif in our six datasets and selected a score threshold of 0.7 to have a large score window. We further filtered the detected motifs for $95 \%$ similarity with the reference matrix of the motif (supplemental data). In a second run, we decreased the similarity threshold to $80 \%$, to investigate if a lower similarity might be properties of protogene motifs.

\subsection{UTRs analyses}

Each de novo transcribed ORF was mapped to its corresponding spliced transcript. The direction of the transcription (forward or reverse) was assessed, and accordingly the 5' and 3' UTRs were retrieved and stored in FASTA format. In order to compare the UTRs of proto-genes with the ones of established human genes, the coordinates of the UTRs of established human genes were extracted from the respective GTF file, and their corresponding FASTA sequences were downloaded from the human reference genome (GRCh38). 
The size and GC content of UTRs were calculated for established human genes as well as for protogenes. The structural and 2-dimensional properties of the 5' UTRs were analysed with ViennaRNA software [61]. The best minimum free energy structure was calculated for all 5' UTRs with the RNA fold function, which implements the algorithm of [62], yielding a single optimal structure.

We further predicted the equilibrium pair probabilities using the command "RNAfold -p -MEA", which calculates base pair probabilities in the thermodynamic ensemble. This function allows us to access the frequency of the minimum free energy (MFE) structure, that corresponds to the probability of a structure to occur according to the Boltzmann weighted ensemble of all structures. The values of ensemble diversity were detected with a python script and correspond to the average Base pair distance between all structures in the thermodynamic ensemble. We calculated the structure probability and the ensemble diversity of 5'UTRs of all proto-genes and compared it to the probability and diversity of 5' UTRs of all human genes and ncRNA. The ncRNAs do not contain UTRs, so we instead calculated the secondary structure of the entire sequences of the ncRNA sequences, by selecting those whose sizes ranged in the same sizes of the 5'UTR of de novo and established human genes.

\subsection{Domain annotation}

To compare the proteins encoded by proto-genes with proteins encoded by established human genes, we downloaded the 20401 human genes from the database Ensembl and their corresponding transcripts. Annotated isoforms were analysed and the longest isoforms were kept to extract protein sequence.

The Pfam database and the pfam_script 'scan.pl' with default parameters [63] were used to annotate domains in both proto-proteins and established human proteins. For all proto-proteins, the results were sorted according to the age class and the genomic position of the proto-genes coding for the respective proteins.

A Gene Ontology (GO) term enrichment was carried out with topGO package in R [64]. Domains were annotated with the 'pfam2go' mapping of Pfam domains to GO terms [65]. The enrichment analysis was done using the ontologies of 'Molecular function'and 'Biological process' with topGOs 'weight01' algorithms. Significantly enriched $(P$-value $\leq 0.05)$ GO terms are visualized as tag clouds.

For detection of novel domains in the studied proto-proteins, the software SEG-HCA was used [66]. The software divides protein sequences by gathering the regions with strong hydrophobic residues and linkers. The highly hydrophobic clusters are known to give rise to secondary structures, which may putatively evolve in new protein domains. The oldest proto-proteins (I5) were not analysed 
regarding their hydrophobic clusters, as they contain annotated domains which may bias the analysis.

\subsection{Statistical and bioinformatic analyses}

All data extractions and analyses were performed with python. The developed scripts are freely available at https://github.com/AnnaGrBio/De_novoGenes_NewGenomicSignals. Statistical analyses and graphs were performed in R (R Core Team; 2017). Since all datasets contained between 500 and 30000 values, shapiro tests were not necessary to test normality. According to the equality of the variances (fisher test), we used welch student test and standard two sample student test for parametric two sample assessment. Anova or Kruskal-Wallis test were used for multiple sample test comparisons. Linear regressions were used by considering the age classes as time variations.

\section{Results}

A total of 27550 human proto-genes were retrieved from the dataset created by [33]. We only considered putative de novo genewith a minimum expression strength of Reads Per million Mapped reads $(\mathrm{RPM})>0.5$. We found 4415 ORFs from proto-genes which began or ended with an unusual codon or which were not assigned to the correct genomic position. The corresponding proto-genes were excluded from the analysis (except for the domain analyses), giving rise to a total number of 23135 human proto-genes.

\subsection{Introns}

\subsubsection{Introns in proto-genes}

The number of proto-genes containing introns was calculated, and these numbers were compared according to the genomic position and age class of the proto-gene (Figure 1). Contrary to our expectations, the number of proto-genes containing introns was surprisingly high, as introns were found in 15710 of the 23135 proto-genes (67.9\%). According to their age class, 75 to $100 \%$ of "exonic" proto-genes possess at least one intron (Figure 1a). The proportions are lower for the other two genomic positions, and vary between 10 to $30 \%$ for "intronic" proto-genes, and between 2.6 to $30 \%$ for "intergenic" proto-genes. For each genomic position, we observed an increase in the proportion of proto-genes containing introns with older age class of the proto-gene. The older a proto-gene is, the more probable it is to contain at least one intron (Table 1). 
Table1

\begin{tabular}{|c|c|c|}
\hline Age class & Genomic position & $\begin{array}{l}\text { mean proportion of proto-genes } \\
\text { with intron }\end{array}$ \\
\hline I0 & “exonic” & 71.4 \\
\hline $\mathrm{I} 1$ & “exonic" & 97.4 \\
\hline I2 & “exonic" & 84 \\
\hline I3 & “exonic” & 89.5 \\
\hline I4 & “exonic” & 90.7 \\
\hline I5 & “exonic" & 98 \\
\hline I0 & "intronic" & 11.6 \\
\hline $\mathrm{I} 1$ & "intronic" & 24.25 \\
\hline I2 & "intronic" & 32.4 \\
\hline I3 & "intronic" & 31.9 \\
\hline I4 & "intronic" & 33.95 \\
\hline I5 & "intronic" & 32.2 \\
\hline I0 & "intergenic" & 3.2 \\
\hline I1 & "intergenic" & 5.5 \\
\hline $\mathrm{I} 2$ & "intergenic" & 5.23 \\
\hline I3 & "intergenic" & 6.22 \\
\hline I4 & "intergenic" & 8.8 \\
\hline I5 & "intergenic" & 30.8 \\
\hline
\end{tabular}

Putative proto-genes contain significantly less introns than established human genes (established genes: 6.57; proto-genes: 5.72; student test p-value < 2.2e-16) (Supplemental data). For all age classes, the average number of introns is higher in "exonic" proto-genes compared to "intronic" and "intergenic" proto-genes (Figure 1b). Comparing the average number of introns in "intronic" and “intergenic" putative de novo genes, we found fewer introns in "intergenic" putative de novo genes for age classes I0 to I4 (Table 2).

Table2. Comparison of the number of introns in proto-genes

\begin{tabular}{|c|c|c|c|}
\hline Age class & Genomic position 1 & Genomic position 2 & p-value t.test \\
\hline I0 & "exonic" & "intronic" & $5.602 \mathrm{e}-07$ \\
\hline I0 & "exonic" & "intergenic" & $5.362 \mathrm{e}-08$ \\
\hline I0 & "intronic" & "intergenic" & $2.2 \mathrm{e}-16$ \\
\hline I1 & "exonic" & "intronic" & $1.972 \mathrm{e}-05$ \\
\hline
\end{tabular}




\begin{tabular}{|c|c|c|c|}
\hline I1 & "exonic" & "intergenic" & $6.549 \mathrm{e}-07$ \\
\hline I1 & "intronic" & "intergenic" & $1.469 \mathrm{e}-15$ \\
\hline I2 & "exonic" & "intronic" & $2.928 \mathrm{e}-05$ \\
\hline I2 & "exonic" & "intergenic" & $1.269 \mathrm{e}-08$ \\
\hline I2 & "intronic" & "intergenic" & $2.2 \mathrm{e}-16$ \\
\hline I3 & "exonic" & "intronic" & $1.995 \mathrm{e}-09$ \\
\hline I3 & "exonic" & "intergenic" & $2.2 \mathrm{e}-16$ \\
\hline I3 & "intronic" & "intergenic" & $2.2 \mathrm{e}-16$ \\
\hline I4 & "exonic" & "intronic" & $2.2 \mathrm{e}-16$ \\
\hline I4 & "exonic" & "intergenic" & $2.2 \mathrm{e}-16$ \\
\hline I4 & "intronic" & "intergenic" & $2.2 \mathrm{e}-16$ \\
\hline I5 & "exonic" & "intronic" & $2.2 \mathrm{e}-16$ \\
\hline I5 & "exonic" & "intergenic" & $2.2 \mathrm{e}-16$ \\
\hline I5 & "intronic" & "intergenic" & $7.034 \mathrm{e}-07$ \\
\hline
\end{tabular}

For "intergenic" and "exonic" proto-genes, the average number of introns increases significantly with gene age. This increase is much stronger in the "exonic" proto-genes than in the "intergenic" ones ("exonic" slope $\mathrm{lm}=0.5042735, \mathrm{~lm}=0.20548$ without I5, "intergenic" slope $\operatorname{lm}=0.256265$ ), but remains low and stable in proto-genes found in the genomic position "intronic" (slope $\operatorname{lm}=$ 0.02916013).

Among the 15710 proto-genes with introns and at least 2 exons, 2148 were found to have the regarding ORF located on one single exon (Figure 1c). The proportion of ORFs found in one single exon is higher for proto-genes with the genomic positions "intergenic" (60.16\%) and "intronic" (63.36 \%) than for "exonic” (36.14 \%). Moreover, taking the gene age into consideration, we found a lower proportion of ORFs located on one single exon compared to ORFs spread over multiple exons with increasing age in all genomic positions ("exonic": from 37.78 \% (I0) to 3.93 \% (I5); “intergenic”: from 76.19 \% (I0) to 18.12 (I5); "intronic”: from 73.45 \% (I0) to 49.24 (I5); Figure 1d).

\subsubsection{Origin of introns in "intergenic" proto-genes}

To investigate the origin of introns in "intergenic" proto-genes, 500 randomly chosen introns from “intergenic" proto-genes were compared via BLASTn with a dataset of established human introns. 205 of the 500 de novo introns were found to have a reasonable hit with at least one established human intron (41\%). All other tested de novo introns (59 \%) did not match with annotated introns, 
suggesting that they emerged by intronisation. Among the 205 de novo introns also found in established human genes, 37 were found in transcripts which ORF was located on one single exon.

\subsection{Motifs upstream proto-genes}

\subsubsection{Average number of motifs from "MotifsAllUpstream}

We searched for 746 motifs from the databases "MotifsAllUpstream” (see method) in six datasets of sequences (from different genomic locations: 200 bp usptream of proto-genes, 200 bp upstream established genes, 200 bp upstream of pseudogenes, 200 bp upstream of ncRNA, 200 bp in randomly selected intergenic regions, $200 \mathrm{bp}$ in randomly selected introns of established genes). For the motifs found upstream of proto-genes, the results were analysed according to the different age classes and genomic positions of proto-genes. A motif is considered as present if it shows $80 \%$ identity to its referent position weight matrix (see methods). Results for a more a common threshold of $95 \%$ are available in Supplemental data, and show significantly the same trends.

Surprisingly, the average number of motifs ("MotifsAllUpstream”) per sequence is lower in the upstream region of established genes compared to upstream region of proto-genes (Welch t.test, pvalue $=2.2 \mathrm{e}-16$ ), with the latter showing a lower average number of motifs compared to the four other datasets (pseudogenes, ncRNA, intergenic regions, introns) (Figure 2a) (Welch t.test, p-value $=2.2 \mathrm{e}-16$ for the four comparisons). We investigated the distribution of the 60 most common motifs over the six datasets (Figure 2b). For 56 of the 60 motifs we either observed the lowest number of motifs for established genes, followed by the dataset of proto-genes, and highest number for the four other categories, or we observed the exact contrary: highest number of motifs for established genes, followed by the dataset of proto-genes, and lastly the four other categories with lower number of motifs.

The database "MotifsAllUpstream" contains both motifs from promotors (except core promotor) and enhancers. In order to distribute the motifs in these two categories, we studied their distribution in 200 bp upstream of all human genes. The motifs most present 200 bp upstream of established genes were considered specific for promotors. The remaining ones are considered specific for enhancers (or distal promotors). We accordingly partitioned the database "MotifsAllUpstream” into two databases, that we call "AllPromoter" and "Enhancer". We calculated the average number of motifs from "AllPromotor" and "Enhancer" in the region 200 bp upstream of proto-genes, according to their age class and genomic position.

Interestingly, proto-genes from the genomic position "exonic" have more motifs specific to the “AllPromotor” dataset, than proto-genes from “intronic” and "intergenic” positions (Welch t.test, pvalue $=2.2 \mathrm{e}-16$ for the two tests) (Figure 2c). On the contrary, the "intergenic" and "intronic" 
proto-genes have more motifs specific from "Enhancer” compared to “exonic” proto-genes (Welch t.test, $\mathrm{p}$-value $=2.2 \mathrm{e}-16$ for the two tests). These results were not affected by the age class of protogenes, except for motifs from the database “AllPromotor" found in "exonic" proto-genes. In this specific case, the average number of motifs is higher for "I5" proto-genes than for "I0" proto-genes (Welch t.test, p-value $=0.000656$ ).

As "intergenic" and "intronic" proto-genes are enriched in enhancers motifs compared to "exonic" proto-genes, we also wondered if this enrichment was higher than expected by chance. We therefore compared the average number of motifs from the "AllEnhancer" database found in "intronic" and "intergenic" proto-genes, to the average number of the same motifs found in the 20000 non-coding regions randomly selected. We discovered that "intergenic" and "intronic" proto-genes are significantly enriched in enhancer motifs compared to non-coding regions, with "intergenic" protogenes being most enriched (Mean motifs "intergenic" proto-genes: 330.7; mean motifs "intronic" proto-genes: 319.8, mean motifs non-coding regions: 305.8; t.test "intergenic" proto-genes vs noncoding region: p.value=2.2e-16; t.test "intronic" proto-genes vs non coding-region: p.value=1.579e08). We investigated the function of the 50 most frequents enhancer motifs found upstream "intergenic" proto-genes. The large majority of these motifs $(48 / 50)$ were found to be associated to homeo genes, which are well known to be involved in development (supplemental data).

\subsubsection{Distribution of "MotifsCore" motifs}

We searched for the nine motifs of the database "MotifsCore" (specific from core promotor), in six datasets of sequences (200 bp surrounding transcription starting point of proto-genes, established genes, pseudogenes, ncRNA, and 200 bp in randomly selected intergenic regions and introns of established genes).

The average number of transcription factor motifs from "MotifsCore" database is highest in promotor regions of established genes (mean $=34.64)$ compared to promotor regions of proto-genes (mean $=32.20$; t.test p-value 2.2e-16), and highest in promotor regions of proto-genes than in the four other datasets (mean ncRNA 29.97; mean pseudogenes 30.06; mean intron 29.79; mean noncoding region 29.97; t.test, p-value 2.2e-16 for the 4 tests; Figure 2d).

Next, we investigated if the average number of motifs from the "MotifsCore" database was divergent according to the age class and the genomic position of proto-genes (Figure 3). We observed that proto-genes at the genomic position "exonic" shown an enriched number of motifs compared to "intronic" and "intergenic" proto-genes (Welch t.test with average motif numbers, pvalue < 2.2e-16). Meanwhile, no significant differences in the numbers of motifs of "intronic" and “intergenic" proto-genes were found (Welch t.test for average motif numbers, $\mathrm{p}$-value $=0.2962$ ), for 
all age classes. By comparing motif numbers of "exonic" proto-genes with motif numbers of established genes, we found that "exonic” proto-genes contain less core motifs in age class I0 than establish genes (32.8 vs 33.6) but this difference is not significant. In age class I5, the average number of motifs in "exonic" proto-genes is similar to the average number of motifs found in established genes (33.5 vs 33.6).

The observed data did not follow a normal distribution (shapiro tests, p-value 2.2e-16). We observe two interesting phenomenon's when investigating the motif distribution in regions upstream of proto-genes. First, in the upstream region of "exonic" proto-genes, we found the density curve showed two different peaks for proto-genes I0, I1, I2, I3 and I4. These results suggest that the "exonic" proto-genes in these age classes may fall into two categories, one being more enriched in motifs, and the other one comprising significantly fewer. Secondly, in the "intergenic" proto-genes from age classes I2, I3, I4 and I5, we observed a bulge in the curve of distribution, suggesting the same conclusion.

Finally, we calculated the percentage of proto-genes which had each of the 9 motifs presents in their promotor, for each age and genomic positions. As expected from previous results, "exonic" protogenes have in average a higher percentage of all the motifs the motifs compared to "intronic" and "intergenic" proto-genes (Figure 4). However, we found an opposite trend with the TATA box motif.

\subsection{UTRs of proto-genes}

\subsubsection{Size and GC content of UTRs}

5' UTRs and 3' UTRs of human proto-genes were compared to those of established human genes. We observed that the size of the 5' UTRs tend to be longer in the youngest proto-genes (I0) compared to established human genes, and become shorter with age (Supplemental data).

We found an average GC content of $59.26 \%$ in the 5' UTR of the human established genes, which is consistent with the literature [67]. The average GC content in the 5' UTRs of proto-genes was $53.23 \%$ on average, and is significantly lower than in established genes(Welch t.test p-value 2.2e16), (Figure 5a).

For 3' UTRs we find the opposite tendency: the GC content of 3' UTRs of established genes is significantly lower (mean: 45.20442), compared to 3' UTRs of proto-genes (mean: 47.1894, t.test p-value 2.2e-16; Figure 5b).

We observed that the GC content was significantly higher in the 5' UTRs of “exonic” proto-genes compared to "intronic" and "intergenic" proto-genes for all age classes (t.test, p-value = 2.2e-16 for the two tests) (Figure 5c). Moreover, the average GC content did not vary between ages classes for "intronic" and "intergenic" proto-genes, whereas the GC content of "exonic" proto-genes is 
significantly higher in oldest proto-genes than in younger proto-genes (Welch t.test, p-value $=$ 0.005916).

\subsection{2. $2 D$ structure of the 5' UTRs}

We studied the secondary structure of the 5' UTRs of human proto-genes, established human genes, and ncRNA. We compared the energy of the structures between proto-genes and established genes, to see if the UTRs of proto-genes are less or equaliy stable as the ones of established genes. Moreover, we compared the diversity and the probability of secondary structures of proto-genes, established genes, and ncRNAs.

The absolute value of the minimum free energy of the thermodynamic ensemble of all 5' UTRs decreases with the size of the 5' UTRs, indicating a higher stability of the structure (Figure 6a left). Most of the 5' UTRs sizes ranged between 0 and 200 bp. The longer a sequence is, the less energetic it is in established genes compared to proto-genes (Figure 6a; lm model, established genes: intercept: 6.778844, slope: -0.405995; proto-genes: intercept: 5.0493149, slope: -0.3385567). We observed that the structure prediction frequencies (structure probability) are significantly higher for 5' UTRs of established genes compared to 5' UTRs of proto-genes (t.test, p-value < 2.2e-16), but interestingly, the structure prediction frequency is higher in proto-genes compared to ncRNA (t.test, p-value < 2.2e-16) (Figure 6b). These results indicate that 5' UTRs of proto-genes are less likely to fold compared to established 5' UTRs, but more likely to fold than ncRNA sequences. Unexpectedly, the structure prediction frequency is higher in the 5' UTR of "intronic" proto-genes $($ mean $=0.15)$ and "intergenic" proto-genes $($ mean $=0.15)$ than in "exonic" proto-genes $($ mean $=$ 0.10) (t.test "exonic" vs "intergenic", p-value $=2.2 \mathrm{e}-16$; t.test "exonic" vs "intronic", p-value = 2.2e-16; t.test "intronic" vs "intergenic", p-value = 0.6133). Additionally, we found that the average diversity value, which indicates the instability of a predicted structure, was significantly higher in proto-genes than in established genes (t.test, p-value $<2$.2e-16), but was significantly lower in 5'UTRs of proto-genes than in ncRNA (t.test, p-value < 2.2e-16; Figure 6c and 6d). Therefore, 5' UTRs of de novo genes are less stable than 5' UTRs of established genes, but more stables than ncRNA. We also observed that the probability of the structure was higher in "exonic" proto-genes $($ mean 62.26$)$ than in "intronic" proto-genes $($ mean $=54.85)$ and "intergenic" proto-genes $($ mean $=$ 55.84) (t.test "exonic" vs "intergenic", p-value = 3.181e-09; t.test "exonic" vs "intronic", p-value = 5.074e-12; t.test "intronic" vs "intergenic", p-value $=0.4401)$. The tendencies on structure probability and diversity (stability) therefore complement each other for all analysed gene/RNA classes and genomic locations: the more likely it is for an UTR to have a structure the more stable it seems to be. 


\subsection{Domains in proto-genes}

\subsubsection{Annotated domains}

We compared the absolute number of Pfam domains found in established human proteins and human proto-proteins. 11244 established human genes contained domains (55\%), while only 7223 proto-proteins contained at least one annotated domain (26\%).

We then investigated if the age and the genomic position of proto-genes are correlating with the presence of annotated domains in the corresponding proto-protein. Independent of the genomic position (“exonic”, “intronic”, "intergenic”), we did not find any known domains in proto-proteins at the ages I0, I1 and I2. Only $0.07 \%$ of proto-proteins contain an annotated domain in age class I3, and $0.22 \%$ in age class I4 (supplemental data). More than 99,7 \% of the domains found in protoproteins are in fact present in the oldest age class I5, which are shared with mouse (Figure 7a). Half of the proto-proteins from the age class I5 (49.02\%) contain an annotated domain. $96.30 \%$ of them are located at the genomic position "exonic" (supplemental data). We still found 185 protoproteins from I5 and genomic position "intronic", and 82 proto-proteins from I5 appeared in the genomic position “intergenic”, containing at least one annotated domain. Taken together, these results indicate that more than $98 \%$ of all proto-proteins that possess a known domain are the oldest proto-proteins (I5; shared with mouse) and are overlapping with an exon. $99.96 \%$ of proto-proteins which are younger than I5 and come from the genomic position "intronic" or "intergenic" do not contain any known domain.

\subsubsection{Novel domains in proto-proteins}

Except for the oldest proto-genes (I5) overlapping with an exon (“exonic”), most proto-proteins did not contain any known domain. We hypothesise that proto-proteins become functional over time by gaining novel domains. We used Seg_HCA [66] to search for hydrophobic clusters as an indicator for domains in proto-proteins from the age classes I0 to I4. The oldest age class I5 was excluded from the analysis, to avoid a bias due to the presence of known domains. For each proto-protein, we retrieved the number of hydrophobic clusters found in the sequence, as well as their size. We found hydrophobic clusters in proto-proteins of all studied age classes (I0-I4, Figure 7b). The number of hydrophobic clusters is significantly lower in the age class I0 than in all other age classes (Welch t.tests (I0-I1; I0-I2; I0-I3; I0-I4, p-values between 2.2e-16 and 8.238e-09). The number of hydrophobic clusters is also significantly lower in I2 and I4 compared to I5 (Welch t.tests, p-value1 $=0.0022$; p-value $1=0.00046)$. The average number of hydrophobic clusters increases with age (linear model, slope $=0.1732102$, mean I0 $=1.300525 ;$ mean I1 $=1.473735 ;$ mean I2 = 1.5208 ; mean I3 = 1.47424 ; mean I4 = 1.573866). 
The average number of hydrophobic clusters is higher in proto-proteins from the genomic position "exonic" $($ mean $=2.52)$ than from the genomic positions "intronic" $($ mean $=1.44$; welch t.test pvalue $=2.2 \mathrm{e}-16$ ) and "intergenic" (mean $=1.34$; welch t.test $\mathrm{p}$-value $=2.2 \mathrm{e}-16$ ) for all analysed age classes (Figure 7c). This was observed for any age class (statistical tests are shown in supplemental data). "Intronic” proto-proteins have more hydrophobic clusters than "intergenic” proto-proteins (Welch t.test, p-value $=1.526 \mathrm{e}-10$ ), but these differences are only significant for age classes I2 and I4. The difference between number of hydrophobic clusters in "intergenic" and "intronic" protogenes was lower compared to "exonic" proto-proteins (statistical tests are shown in supplemental data).

\section{Discussion}

In the present manuscript, we investigated four different gene properties of 23135 human protogenes, which are; presence of introns, presence of motifs, characteristics of UTRs and domain coverage. All proto-genes were investigated according to their genomic position and their age.

\subsection{Introns}

In 2011, [39] reported that 59 of the 60 de novo genes identified in human are single exon genes. Another experimental study investigated the function of de novo genes and reported that most of the analysed de novo genes contain a single exon [5]. In contrast, we found that half of all human proto-genes of our dataset contain introns. This very unexpected results suggest a more complex structure than previously thought for these new genes, even though proto-genes contain fewer introns than established genes. We found that "exonic" putative proto-genes contain on average more introns than "intergenic" and "intronic" proto-genes. A possible explanation would be that “exonic” proto-genes not only overlap with parts of an established gene but copied introns of the established gene into their own sequence. Our finding that about $50 \%$ of the analysed proto-genes contain introns, indicates that splicing of proto-genes surprising already occurs even in the youngest proto-genes. This would even explain why [33] found multiple transcripts per ORF with differing lengths during their transcriptome assembly.

Furthermore, we discovered that $13.7 \%$ of the analysed human proto-genes containing introns have the ORFs located on one single exon. Such a structure is uncommon but has already been documented in the de novo gene atlas [68]. The coding sequence of atlas is located on the first of the two exons presents in the gene. Our study suggests that this structure is more common than expected in de novo genes. ORFs being located on one single exon are more common in young than in old proto-genes. It has already been reported that de novo ORFs increase in length over time [33], 
which could explain why small ORFs located on one single exon evolve to be overlapping with several exons by gaining mutations.

We found that the average number of introns increases with age for all proto-genes. This result suggests that proto-genes acquire introns over time. Seven scenarios are known to describe intron emergence in genes [38]: Intron transposition, transposon insertion, tandem duplication, intron gain during double strand break repair, insertion of a group2 intron, intron transfer, and intronisation. Interestingly, $41 \%$ of the introns from a subset of 500 randomly picked introns from "intergenic" de novo transcripts had a BLAST hit with established introns from the human reference genome. To the best of our knowledge, the origin of introns in de novo genes has never been studied before, and a deeper investigation is warranted for the future. The fact that $41 \%$ of the investigated introns have a dectable signal of homology using BLAST to existing introns suggest that proto-genes captured existing introns. It would be interesting to focus on the putative role that transposable elements may have played in proto-gene emergence. It has already been shown that orphan genes can emerge via the exaptation of transposable elements [6]. In 2021, [69] discovered that 37 orphan genes in the genome of Oryza sativa had emerged via recruitment of transposable elements. The presence of introns in "intergenic" proto-genes could also be an interesting indicator that de novo genes and/or their introns emerged via the movement of a transposable element.

\subsection{Motifs}

By comparing four datasets of sequences to upstream regions of established human genes and human proto-genes, we demonstrated that "exonic" proto-genes are significantly enriched in motifs which are characterisitc for promoters and core promoters. The average number of motifs specific for promotors found upstream of "exonic" proto-genes still remains lower than the number of promotor motifs found upstream of established human genes, except for oldest "exonic" protogenes. No difference was found between the average number of core promotor motifs of established human genes and "exonic" proto-genes at the age class I5. As these old proto-genes overlap with human established genes, this finding suggests a reuse of pre-existing regulatory sequences in old proto-genes [70]. In 2015, [71] observed an enrichment in transcription factor binding sites in the promotor regions of the 2,714 proto-genes that they identified in human and chimpanzee. $20 \%$ of these proto-genes matched with long ncRNAs, half of the dataset overlapped with exons and partly with introns, which may have biased the results. The observed increase of transcription factor binding sites in core promotor motifs with age of "exonic" proto-genes may indicate a gain of motifs with age. The "exonic" proto-genes are the most complicated to characterise, as they may or may not overlap with the promoter and can also be transcribed in the reverse direction. 
On the other hand, we demonstrated that "intronic" and "intergenic" proto-genes are specifically enriched in enhancer motifs, and that their average number of enhancer motifs is higher than in randomly selected non-coding regions. This means that their proximity to these motifs might be a condition for their pervasive existence. The enrichment of enhancer motifs in proto-genes compared to non-coding regions, however, does not change with age, corroborating their "sine qua non" status.

The fact that putative "intergenic" and "intronic" proto-genes contain more enhancer than promotor motifs is an interesting finding. One of the reasons could be that non-coding regions, such as intergenic and intronic regions, may be translated through existing enhancers rather than gaining their own regulatory motifs. This result confirms the finding of [72], who demonstrated that young de novo genes in mice emerge closer to enhancers than to promotors. They assumed that de novo transcribed ORFs, which emerge in non-coding regions may be transcribed due to the facilitated environment created by the DNA loop. They also found that older de novo genes were in closer proximity to promotors than to enhancers. In contrast to this, we did not find any significant change with gene age. However, [72] did not distinguish between "intergenic" and "exonic" de novo genes except for the youngest age class, which makes the studies not entirely comparable.

Not only do "intergenic" and "intronic" proto-genes appear more often in regions rich in enhancers, but most of these enhancers are associated to morphogenesis functions. Homeodomain proteins are highly expressed [73], and can regulate the expression of many genes [74]. The combination of these two properties could explain that any new ORF which emerges close to homeotic transcription factors has a higher chance to be expressed and maintained throughout evolution.

The average number of motifs specific for core promotors found upstream of "intergenic" and "intronic" proto-genes is low and does not significantly differ from non coding-regions. However, when looking at the distribution, we observed subgroups enriched in motifs from the core promotor compared to the remaining of the group. Motifs from the core promotor are more frequent in a subset of "intergenic" proto-genes compared to the average trend. This result might confirm that “intergenic" proto-genes can gain promotor motifs with age. Again, further investigations using a wider range of data sets are warranted to confirm generality of these findings.

Even though "intergenic" and "intronic" proto-genes are not enriched in core promotor motifs, the motif TATA was found to be more present upstream of these proto-genes than upstream of "exonic" ones. Generally, not all established human genes have TATA motifs in their promotor either. According to [75], only $24 \%$ of human genes contain a TATA motif in their promotors. Genes containing a TATA box in their promotor have been shown to have an increased transcriptional regulation compared to genes without a TATA box [76]. In 2013, [77] that genes from S.cerevisiae having an essential TATA box, have a significant codon bias and higher levels of expression. We 
hypothesise that the presence of a TATA box in "intergenic" or "intronic" proto-genes could be maintained by selection, as it allows the expression of the gene. Also, the enrichment in TATA boxes and homeobox transcription factors upstream of "intergenic" and "intronic" proto-genes could be an explanation for their pervasive expression.

\subsection{UTRs}

We investigated the size, GC content, and the predicted free energy of the 2D structure of 5' UTRs in proto-genes and compared it to established genes. 5' and 3' UTRs are longer in proto-genes than in established genes, except for "I5". Until now, studies only focussed on increasing length of de novo ORFs with age, which can be due to the gain of a new start or stop codon in the transcript. In case the length of an ORF is not modified during evolution, the length by which a transcript increases over time correlates with a decrease in length of its 5' UTR. In 2009, [78] showed that mammalian 5' UTRs evolve under weaker selective constraints, giving rise to an accumulation of new AUG codons. In S.cerevisiae, [79] found that the lineages that had a longer 5' UTR showed a substantial decrease of expression correlation with ribosomes. Moreover, [80] observed a correlation between the size of 5' UTR regions and the GC content. They concluded that the size of UTRs may be therefore shaped by structural forces.

We observed that the GC content of the 5' UTRs is significantly lower in proto-genes than in established genes. However, the GC content is higher in "exonic" proto-genes than in "intergenic" and “intronic” proto-genes. A large part of “exonic” proto-genes have a 5' UTR overlapping with established exons, which generally have higher GC contents compared to non-coding regions [81]. Hence, the emergence of de novo genes in regions of high GC content may promote higher levels of structure of the 5' UTR. In contrast, the emergence of new genes overlapping with introns or noncoding regions results in 5' UTRs that have a low GC content.

In a second step, we compared the structure diversity and probability of the UTRs of proto-genes to the same properties of established genes and ncRNA. As expected, the probability of forming stable RNA structure was lower in UTRs of proto-genes than in established genes, and the diversity was higher in proto-genes. However, we observed the opposite trend in ncRNA, indicating a higher probability for in UTRs of proto-genes compared to ncRNAs. This result suggests that the foldability and the structure of 5' UTRs of proto-genes could be prone to selection.

Taken together, proto-genes show a lower GC content, a lower structure probability, and higher amount of discorded regions (structure diversity) compared to established genes but a higher structure probability and lower likelihood for disordered regions than ncRNAs. We therefore hypothesise 5' UTRs of proto-genes to be less likely to fold compared to 5' UTRs of established genes but are more likely to fold compared to ncRNAs. Surprisingly, we found that UTRs of 
“intergenic” and “intronic” proto-genes are more likely to fold properly than UTRs of "exonic” proto-genes. Hence, we found the UTRs of "intergenic" and "intronic" proto-genes to have a low GC content but higher potential for folding. Low GC content is a characteristic of protein binding mRNAs [82]. Also, RNA interference methods have shown that low GC content can increase RNAi efficiency [83]. It is possible that the UTRs of "exonic" proto-genes are constrained to have a high GC content as they overlap with coding exons, wheras intergenic and intronic UTRs are subjected by fewer constrains.

\subsection{Domains}

$26.21 \%$ of the analysed proto-proteins contained annotated domains. Among them, $96.30 \%$ were found in "exonic" proto-genes, and nearly all of them belong exclusively to the oldest outgroup proto-genes (I5). In other words, the majority of all detected known/annotated domains within proto-genes originated at least 90 Mya and share a common ancestor with mouse. Young protogenes do not contain known/annotated domains in their encoded proteins. Furthermore, primate specific “intronic” and “intergenic” proto-proteins do not contain any detectable domains (I0 to I4). We highlight that the absence of annotated domains can be used as a support to distinguish any proto-genes from any other novel gene (emerged by other mechanism than “de novo”).

However, this criterion does not work for old proto-genes. One reason why only I5 "exonic" protogenes contain known domains could be that “exonic" proto-genes are able to gain domains from the exon with which they overlap. A couple of mechanisms have been described to illustrate how proteins can gain domains $[49,84]$. Notably, exon extension allows proteins to gain pre-existing domains [54,85]. This fits to the observation that de novo genes become longer with age [33]. The increase in lenght and the mutations throughout evolution could allow new genes to capture existing domains from their host gene. More interesting is why young (I0 to I4) "exonic" proto-genes never contain domains, although they could utilise a present domain of the overlapping exon. This could be due to destabilising effects of the added sequence parts to the existing domain, rendering it nonfunctional [86]. In 2013, [54] used Pfam domains to investigate their emergence time, dividing them in "mammal specific", "vertebrate specific", and “older domains". In another study, [55] studied novel domains in insects with divergence times between 2 My and $390 \mathrm{My}$. They demonstrated that novel domains could emerge from scratch in terminal regions of proteins.

As a next step, we investigated hydrophobic clusters to identify putative novel domains in protein from proto-genes of age I0 to I4 using SEG-HCA. We observed that the majority of proto-proteins contain hydrophobic clusters. Proteins from proto-genes emerging at the genomic position "exonic" contain more clusters by average than "intronic" and "intergenic" proto-proteins. Moreover, we show that the average number of hydrophobic clusters in proteins increases with age, and this 
increase is even stronger for "exonic" proto-genes. The observation that proto-genes contain hydrophobic clusters is in agreement with a recent study, in which the authors demonstrated that novel domains were present and evolved in yeast de novo genes [87]. Even if domains are conserved as independent units through evolutionary time and are mainly rearranged, novel domains have been shown to emerge regularly in proteins to add specific novel functionality $[52,55]$.

\section{Conclusions}

The four studied properties (introns, transcription motifs, UTRs, and protein domains) show significant differences between proto-genes and established genes, making all of them exciting features to assess the emergence of proto-genes. However, we demonstrate that these properties are not only strongly influenced by gene age, but also by genomic position of the proto-gene. The discovery that genomic positions affect proto-gene's properties is of major importance. Future studies on proto-genes should therefore take the genomic position of proto-genes as well as their age into account.

\section{Supplementary Materials: The following are available online at https://doi.org/10.5281/zenodo.5786006}

Author Contributions: For research articles with several authors, a short paragraph specifying their individual contributions must be provided. The following statements should be used "Conceptualization, A.G. and E.B.B.; methodology, A.G., E.D., K.B; python programs, A.G.; validation, A.G., K.B. and E.D., E.B.B; formal analysis, A.G., K.B, E.D, E.B.B; investigation, A.G., E.B.B; resources, A.G.; data curation, A.G., E.B.B; writing-original draft preparation, A.G.; writing-review and editing, K.B., E.D, E.B.B; visualization, A.G., K.B; supervision, E.B.B; project administration, A.G., E.B.B; funding acquisition, A.G, E.B.B. All authors have read and agreed to the published version of the manuscript."

Funding: AG and EBB acknowledge funding by Alexander von Humboldt-Stiftung. KB is funded by a Deutsche Forschungsgemeinschaft (DFG).

Data Availability Statement: The datasets generated and/or analysed during the current study are available in the github https://github.com/AnnaGrBio/De_novoGenes_NewGenomicSignals, and in the supplemental data in https://doi.org/10.5281/zenodo.5786006

Acknowledgments: We ackowledge Alun Jones for is revision on the paper, Peter Czuppon for his assessment of the statistical tests.

Conflicts of Interest: The authors declare no conflict of interest.

\section{References}

1. Begun, D. J., Lindfors, H. A., Kern, A. D. \& Jones, C. D. Evidence for de Novo Evolution of Testis-Expressed Genes in the Drosophila yakuba / Drosophila erecta Clade. Genetics 176, 1131-1137 (2007).

2. Kaessmann, H. Origins, evolution, and phenotypic impact of new genes. Genome Res 20, 13131326 (2010).

3. Oss, S. B. V. \& Carvunis, A.-R. De novo gene birth. PLOS Genetics 15, e1008160 (2019). 
4. Long, M., Betrán, E., Thornton, K. \& Wang, W. The origin of new genes: glimpses from the young and old. Nat Rev Genet 4, 865-875 (2003).

5. McLysaght, A. \& Guerzoni, D. New genes from non-coding sequence: the role of de novo protein-coding genes in eukaryotic evolutionary innovation. Philos Trans R Soc Lond B Biol Sci 370, 20140332 (2015).

6. Schlötterer, C. Genes from scratch--the evolutionary fate of de novo genes. Trends Genet 31, 215-219 (2015).

7. Schmitz, J. F. \& Bornberg-Bauer, E. Fact or fiction: updates on how protein-coding genes might emerge de novo from previously non-coding DNA. F1000Res 6, 57 (2017).

8. Carvunis, A.-R. et al. Proto-genes and de novo gene birth. Nature 487, 370-374 (2012).

9. Sorek, R. The birth of new exons: Mechanisms and evolutionary consequences. RNA 13, 16031608 (2007).

10. Sabath, N., Wagner, A. \& Karlin, D. Evolution of viral proteins originated de novo by overprinting. Mol Biol Evol 29, 3767-3780 (2012).

11. Rancurel, C., Khosravi, M., Dunker, A. K., Romero, P. R. \& Karlin, D. Overlapping genes produce proteins with unusual sequence properties and offer insight into de novo protein creation. J Virol 83, 10719-10736 (2009).

12. Li, D. et al. A de novo originated gene depresses budding yeast mating pathway and is repressed by the protein encoded by its antisense strand. Cell Res 20, 408-420 (2010).

13. Makałowska, I., Lin, C.-F. \& Hernandez, K. Birth and death of gene overlaps in vertebrates. BMC Evolutionary Biology 7, 193 (2007).

14. Tajnik, M. et al. Intergenic Alu exonisation facilitates the evolution of tissue-specific transcript ends. Nucleic Acids Res 43, 10492-10505 (2015).

15. Schmitz, J. \& Brosius, J. Exonization of transposed elements: A challenge and opportunity for evolution. Biochimie 93, 1928-1934 (2011).

16. Toll-Riera, M. et al. Origin of Primate Orphan Genes: A Comparative Genomics Approach. Molecular Biology and Evolution 26, 603-612 (2009).

17. Cai, J., Zhao, R., Jiang, H. \& Wang, W. De Novo Origination of a New Protein-Coding Gene in Saccharomyces cerevisiae. Genetics 179, 487-496 (2008).

18. Gubala, A. M. et al. The Goddard and Saturn Genes Are Essential for Drosophila Male Fertility and May Have Arisen De Novo. Mol Biol Evol 34, 1066-1082 (2017).

19. Lange, A. et al. Structural and functional characterization of a putative de novo gene in Drosophila. Nat Commun 12, 1667 (2021).

20. Zhuang, X., Yang, C., Murphy, K. R. \& Cheng, C.-H. C. Molecular mechanism and history of non-sense to sense evolution of antifreeze glycoprotein gene in northern gadids. PNAS 116, 4400-4405 (2019).

21. Wang, G. et al. Understanding Transcription Factor Regulation by Integrating Gene Expression and DNase I Hypersensitive Sites. BioMed Research International 2015, e757530 (2015).

22. Li, D., Yan, Z., Lu, L., Jiang, H. \& Wang, W. Pleiotropy of the de novo-originated gene MDF1. Sci Rep 4, 7280 (2014).

23. Zhang, Y. E., Landback, P., Vibranovski, M. D. \& Long, M. Accelerated Recruitment of New Brain Development Genes into the Human Genome. PLOS Biology 9, e1001179 (2011).

24. O’Toole, Á. N., Hurst, L. D. \& McLysaght, A. Faster Evolving Primate Genes Are More Likely to Duplicate. Molecular Biology and Evolution 35, 107-118 (2018).

25. Casola, C. From De Novo to "De Nono": The Majority of Novel Protein-Coding Genes Identified with Phylostratigraphy Are Old Genes or Recent Duplicates. Genome Biology and Evolution 10, 2906-2918 (2018).

26. Slater, G. S. C. \& Birney, E. Automated generation of heuristics for biological sequence comparison. BMC Bioinformatics 6, 31 (2005).

27. Tautz, D. \& Domazet-Lošo, T. The evolutionary origin of orphan genes. Nat Rev Genet 12, 692702 (2011). 
bioRxiv preprint doi: https://doi.org/10.1101/2022.01.04.474757; this version posted January 4, 2022. The copyright holder for this preprint (which was not certified by peer review) is the author/funder. All rights reserved. No reuse allowed without permission.

28. Wilson, B. A., Foy, S. G., Neme, R. \& Masel, J. Young genes are highly disordered as predicted by the preadaptation hypothesis of de novo gene birth. Nat Ecol Evol 1, 1-6 (2017).

29. Vakirlis, N. et al. A Molecular Portrait of De Novo Genes in Yeasts. Mol Biol Evol 35, 631-645 (2018).

30. Li, Z.-W. et al. On the Origin of De Novo Genes in Arabidopsis thaliana Populations. Genome Biol Evol 8, 2190-2202 (2016).

31. Heames, B., Schmitz, J. \& Bornberg-Bauer, E. A Continuum of Evolving De Novo Genes

Drives Protein-Coding Novelty in Drosophila. J Mol Evol 88, 382-398 (2020).

32. Schmitz, J. F., Chain, F. J. J. \& Bornberg-Bauer, E. Evolution of novel genes in three-spined stickleback populations. Heredity 125, 50-59 (2020).

33. Dowling, D., Schmitz, J. F. \& Bornberg-Bauer, E. Stochastic Gain and Loss of Novel Transcribed Open Reading Frames in the Human Lineage. Genome Biology and Evolution 12, 2183-2195 (2020).

34. Jo, B.-S. \& Choi, S. S. Introns: The Functional Benefits of Introns in Genomes. Genomics Inform 13, 112-118 (2015).

35. Will, C. L. \& Lührmann, R. Spliceosome Structure and Function. Cold Spring Harb Perspect Biol 3, a003707 (2011).

36. Irimia, M. \& Roy, S. W. Origin of spliceosomal introns and alternative splicing. Cold Spring Harb Perspect Biol 6, a016071 (2014).

37. Lynch, M. Intron evolution as a population-genetic process. PNAS 99, 6118-6123 (2002).

38. Yenerall, P. \& Zhou, L. Identifying the mechanisms of intron gain: progress and trends. Biol Direct 7, 29 (2012).

39. Wu, D.-D., Irwin, D. M. \& Zhang, Y.-P. De Novo Origin of Human Protein-Coding Genes. PLOS Genetics 7, e1002379 (2011).

40. Pufall, M. A. \& Kaplan, C. D. Mechanisms of eukaryotic transcription. Genome Biology 14, 311 (2013).

41. Londei, P. Evolution of translational initiation: new insights from the archaea $\star$ FEMS Microbiology Reviews 29, 185-200 (2005).

42. Nakagawa, S., Niimura, Y., Gojobori, T., Tanaka, H. \& Miura, K. Diversity of preferred nucleotide sequences around the translation initiation codon in eukaryote genomes. Nucleic Acids Res 36, 861-871 (2008).

43. Kozak, M. Possible role of flanking nucleotides in recognition of the AUG initiator codon by eukaryotic ribosomes. Nucleic Acids Res 9, 5233-5252 (1981).

44. Mignone, F., Gissi, C., Liuni, S. \& Pesole, G. Untranslated regions of mRNAs. Genome Biology 3, reviews0004.1 (2002).

45. Sweeney, R., Fan, Q. \& Yao, M. C. Antisense ribosomes: rRNA as a vehicle for antisense RNAs. Proc Natl Acad Sci U S A 93, 8518-8523 (1996).

46. Gingras, A. C., Raught, B. \& Sonenberg, N. eIF4 initiation factors: effectors of mRNA recruitment to ribosomes and regulators of translation. Annu Rev Biochem 68, 913-963 (1999).

47. Chatterjee, S. \& Pal, J. K. Role of 5'- and 3'-untranslated regions of mRNAs in human diseases. Biol Cell 101, 251-262 (2009).

48. Richardson, J. S. The Anatomy and Taxonomy of Protein Structure. in Advances in Protein Chemistry (eds. Anfinsen, C. B., Edsall, J. T. \& Richards, F. M.) vol. 34 167-339 (Academic Press, 1981).

49. Björklund, A. K., Ekman, D., Light, S., Frey-Skött, J. \& Elofsson, A. Domain rearrangements in protein evolution. J Mol Biol 353, 911-923 (2005).

50. Ren, S. et al. The conservation pattern of short linear motifs is highly correlated with the function of interacting protein domains. BMC Genomics 9, 452 (2008).

51. Bornberg-Bauer, E. \& Albà, M. M. Dynamics and adaptive benefits of modular protein evolution. Curr Opin Struct Biol 23, 459-466 (2013). 
52. Dohmen, E., Klasberg, S., Bornberg-Bauer, E., Perrey, S. \& Kemena, C. The modular nature of protein evolution: domain rearrangement rates across eukaryotic life. BMC Evolutionary Biology 20, 30 (2020).

53. Kleppe, A. S. \& Bornberg-Bauer, E. Robustness by intrinsically disordered C-termini and translational readthrough. Nucleic Acids Research 46, 10184-10194 (2018).

54. Toll-Riera, M. \& Albà, M. M. Emergence of novel domains in proteins. BMC Evolutionary Biology 13, 47 (2013).

55. Klasberg, S., Bitard-Feildel, T., Callebaut, I. \& Bornberg-Bauer, E. Origins and structural properties of novel and de novo protein domains during insect evolution. FEBS J 285, 26052625 (2018).

56. Howe, K. L. et al. Ensembl 2021. Nucleic Acids Research 49, D884-D891 (2021).

57. Karolchik, D. et al. The UCSC Table Browser data retrieval tool. Nucleic Acids Res 32, D493496 (2004).

58. Fornes, O. et al. JASPAR 2020: update of the open-access database of transcription factor binding profiles. Nucleic Acids Research 48, D87-D92 (2020).

59. Butler, J. E. F. \& Kadonaga, J. T. The RNA polymerase II core promoter: a key component in the regulation of gene expression. Genes Dev. 16, 2583-2592 (2002).

60. Cock, P. J. A. et al. Biopython: freely available Python tools for computational molecular biology and bioinformatics. Bioinformatics 25, 1422-1423 (2009).

61. Lorenz, R. et al. ViennaRNA Package 2.0. Algorithms for Molecular Biology 6, 26 (2011).

62. Zuker, M. \& Stiegler, P. Optimal computer folding of large RNA sequences using thermodynamics and auxiliary information. Nucleic Acids Res 9, 133-148 (1981).

63. El-Gebali, S. et al. The Pfam protein families database in 2019. Nucleic Acids Res 47, D427D432 (2019).

64. Alexa, A., Rahnenführer, J. \& Lengauer, T. Improved scoring of functional groups from gene expression data by decorrelating GO graph structure. Bioinformatics 22, 1600-1607 (2006).

65. Mitchell, A. et al. The InterPro protein families database: the classification resource after 15 years. Nucleic Acids Res 43, D213-221 (2015).

66. Faure, G. \& Callebaut, I. Comprehensive Repertoire of Foldable Regions within Whole Genomes. PLOS Computational Biology 9, e1003280 (2013).

67. Zhang, L., Kasif, S., Cantor, C. R. \& Broude, N. E. GC/AT-content spikes as genomic punctuation marks. PNAS 101, 16855-16860 (2004).

68. Rivard, E. L. et al. A putative de novo evolved gene required for spermatid chromatin condensation in Drosophila melanogaster. PLoS Genet 17, e1009787 (2021).

69. Jin, G.-H. et al. Genetic innovations: Transposable element recruitment and de novo formation lead to the birth of orphan genes in the rice genome. Journal of Systematics and Evolution 59, 341-351 (2021).

70. Knowles, D. G. \& McLysaght, A. Recent de novo origin of human protein-coding genes. Genome Res 19, 1752-1759 (2009).

71. Ruiz-Orera, J. et al. Origins of De Novo Genes in Human and Chimpanzee. PLOS Genetics 11, e1005721 (2015).

72. Majic, P. \& Payne, J. L. Enhancers Facilitate the Birth of De Novo Genes and Gene Integration into Regulatory Networks. Mol Biol Evol 37, 1165-1178 (2020).

73. Hirth, F., Hartmann, B. \& Reichert, H. Homeotic gene action in embryonic brain development of Drosophila. Development 125, 1579-1589 (1998).

74. Corsetti, M. T. et al. Differential DNA binding properties of three human homeodomain proteins. Nucleic Acids Research 20, 4465-4472 (1992).

75. Yang, C., Bolotin, E., Jiang, T., Sladek, F. M. \& Martinez, E. Prevalence of the Initiator over the TATA box in human and yeast genes and identification of DNA motifs enriched in human TATA-less core promoters. Gene 389, 52-65 (2007).

76. Bae, S.-H., Han, H. W. \& Moon, J. Functional analysis of the molecular interactions of TATA box-containing genes and essential genes. PLoS One 10, e0120848 (2015). 
77. Han, H. W., Bae, S. H., Jung, Y. H., Kim, J. H. \& Moon, J. Genome-wide characterization of the relationship between essential and TATA-containing genes. FEBS Lett 587, 444-451 (2013).

78. Resch, A. M., Ogurtsov, A. Y., Rogozin, I. B., Shabalina, S. A. \& Koonin, E. V. Evolution of alternative and constitutive regions of mammalian 5'UTRs. BMC Genomics 10, 162 (2009).

79. Lin, Z. \& Li, W.-H. Evolution of 5' untranslated region length and gene expression reprogramming in yeasts. Mol Biol Evol 29, 81-89 (2012).

80. Reuter, M., Engelstädter, J., Fontanillas, P. \& Hurst, L. D. A test of the null model for 5' UTR evolution based on GC content. Mol Biol Evol 25, 801-804 (2008).

81. Leppek, K., Das, R. \& Barna, M. Functional 5' UTR mRNA structures in eukaryotic translation regulation and how to find them. Nat Rev Mol Cell Biol 19, 158-174 (2018).

82. Courel, M. et al. GC content shapes mRNA storage and decay in human cells. eLife 8, e49708 (2019).

83. Chan, C. Y. et al. A structural interpretation of the effect of GC-content on efficiency of RNA interference. BMC Bioinformatics 10, S33 (2009).

84. Nasir, A., Kim, K. M. \& Caetano-Anollés, G. Global Patterns of Protein Domain Gain and Loss in Superkingdoms. PLOS Computational Biology 10, e1003452 (2014).

85. Marsh, J. A. \& Teichmann, S. A. How do proteins gain new domains? Genome Biol 11, 126 (2010).

86. Levy, Y. Protein Assembly and Building Blocks: Beyond the Limits of the LEGO Brick Metaphor. Biochemistry 56, 5040-5048 (2017).

87. Papadopoulos, C. et al. Intergenic ORFs as elementary structural modules of de novo gene birth and protein evolution. Genome Res. 31, 2303-2315 (2021). 
a)

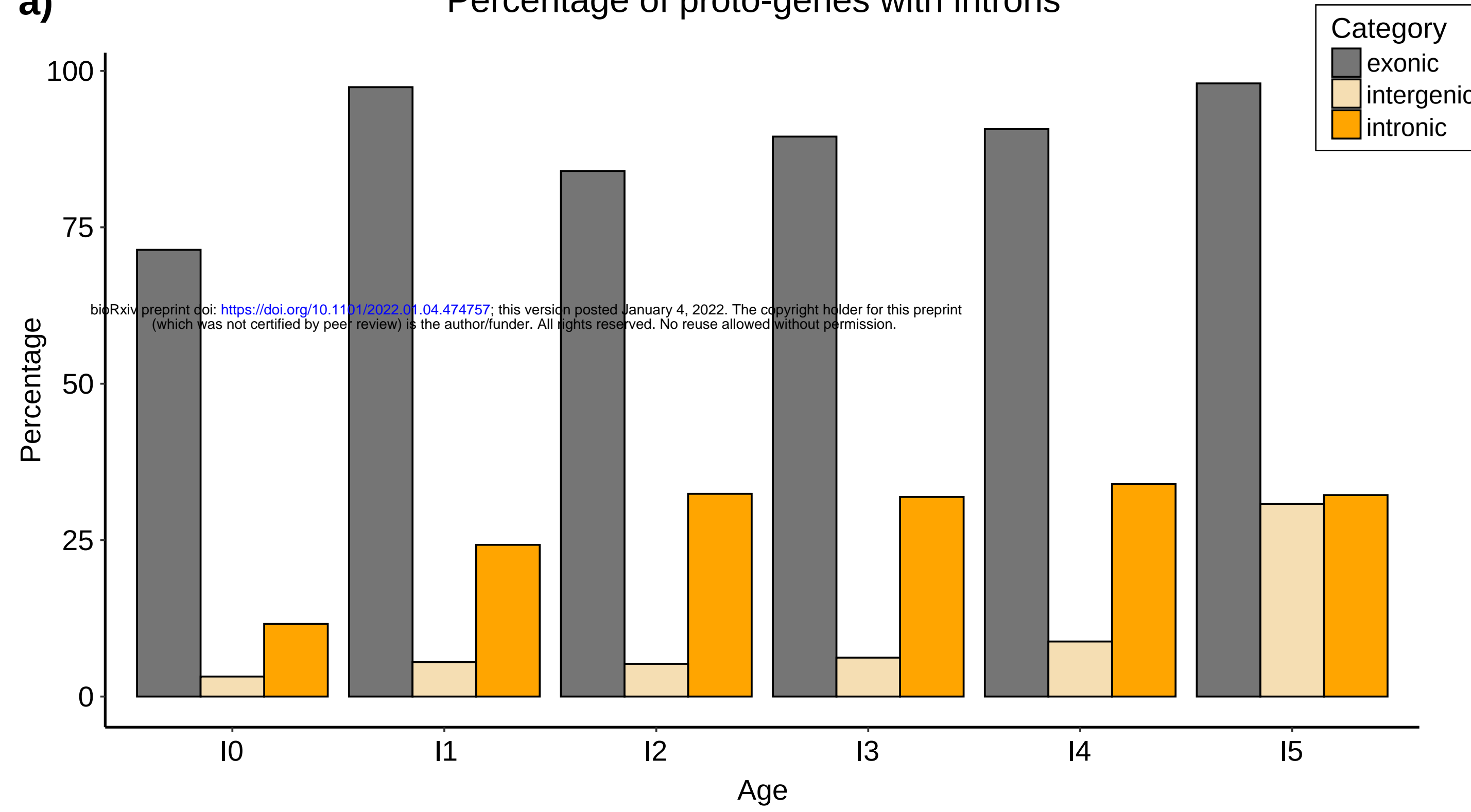

b)

Number of introns per proto-gene

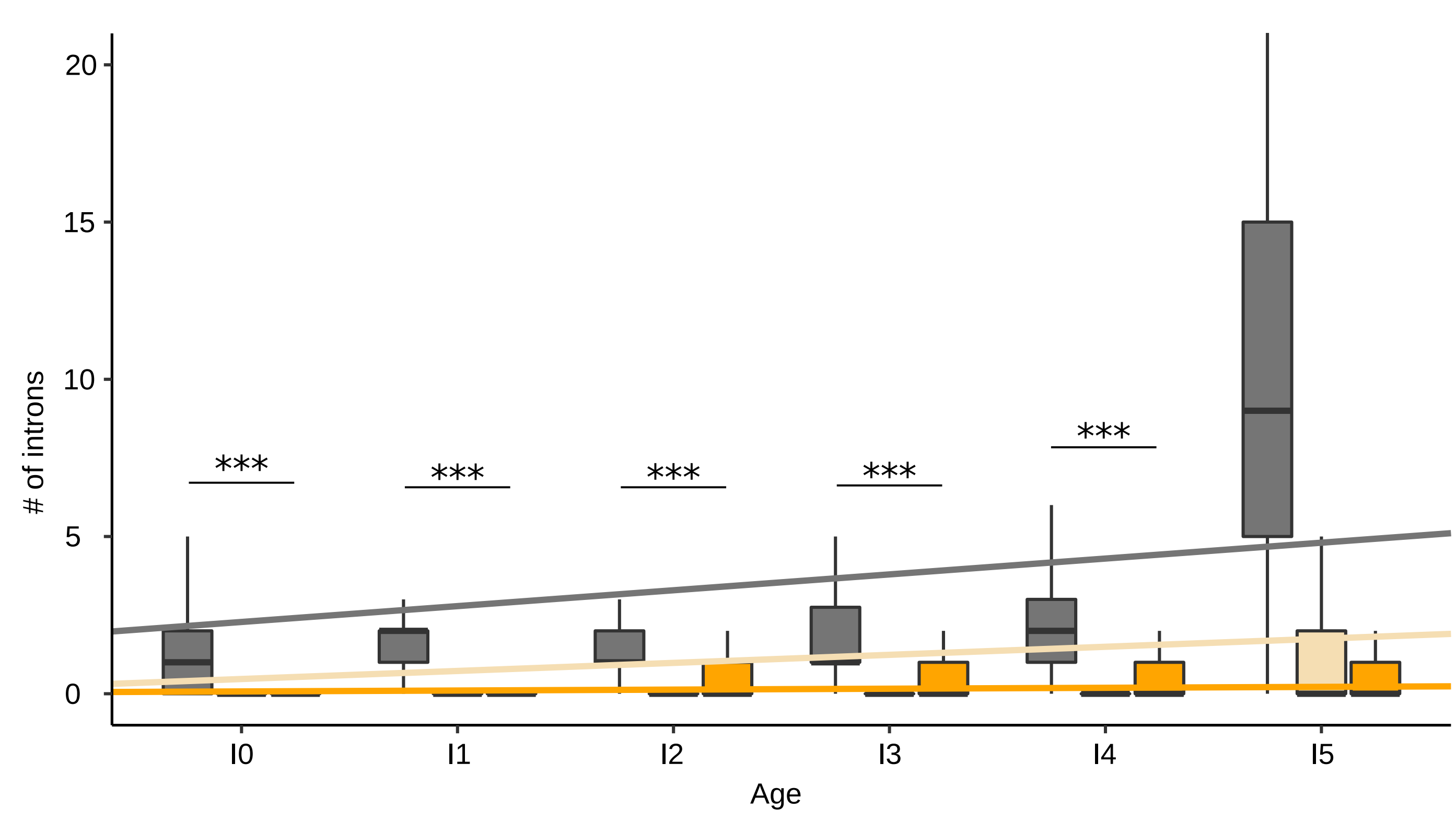

c)

Schematic overview of intron positions
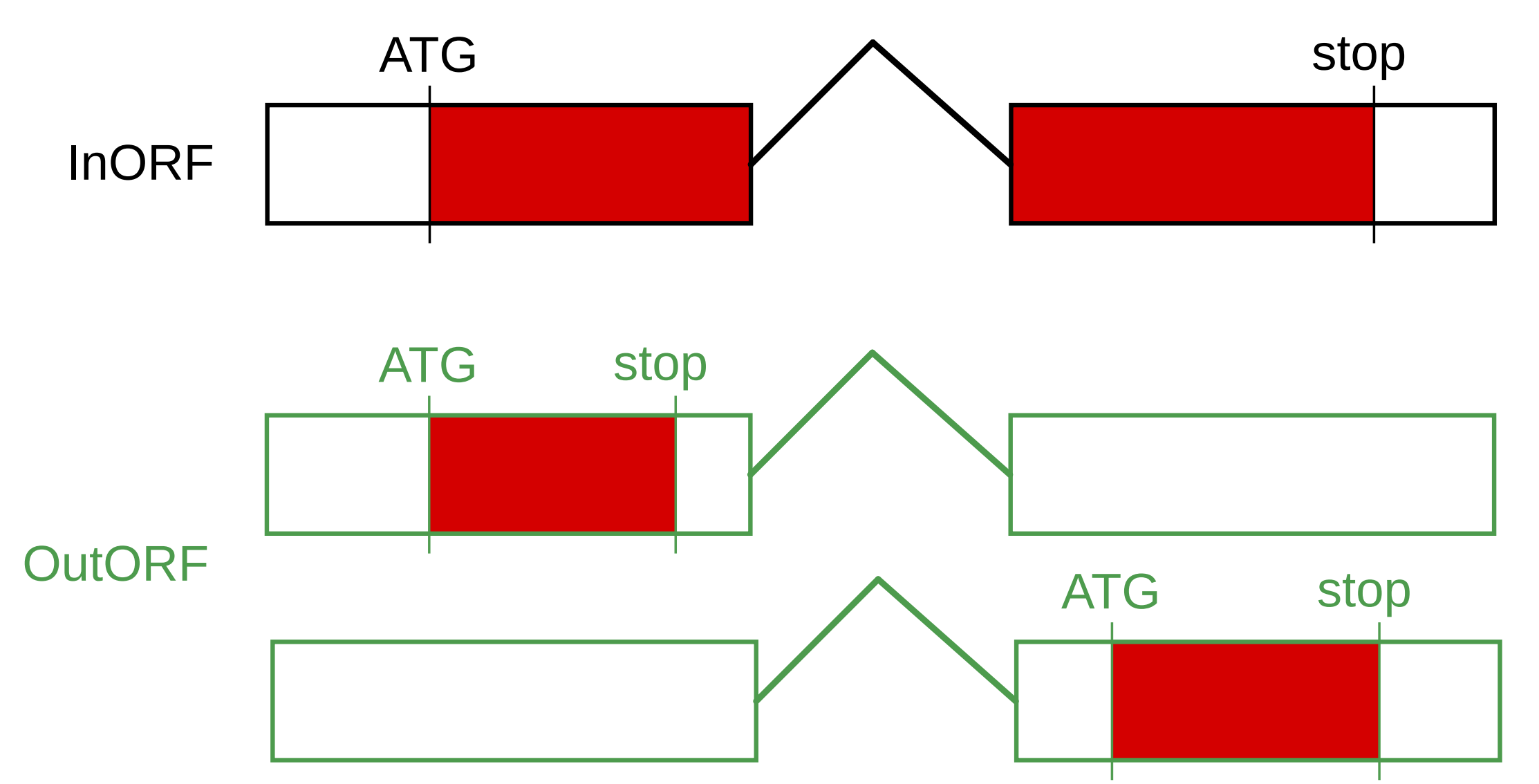

d)

Percentage of introns relative to gene location

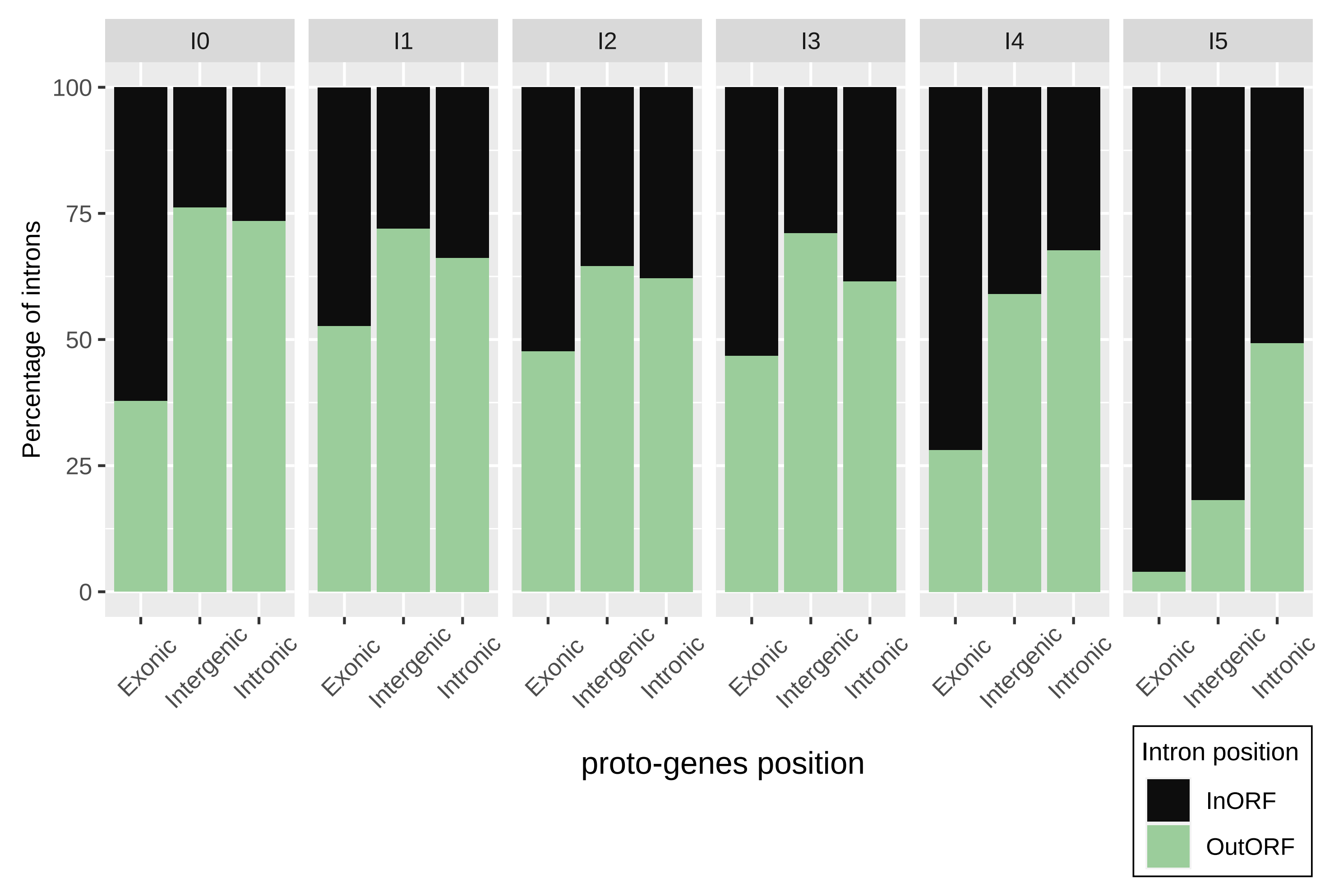





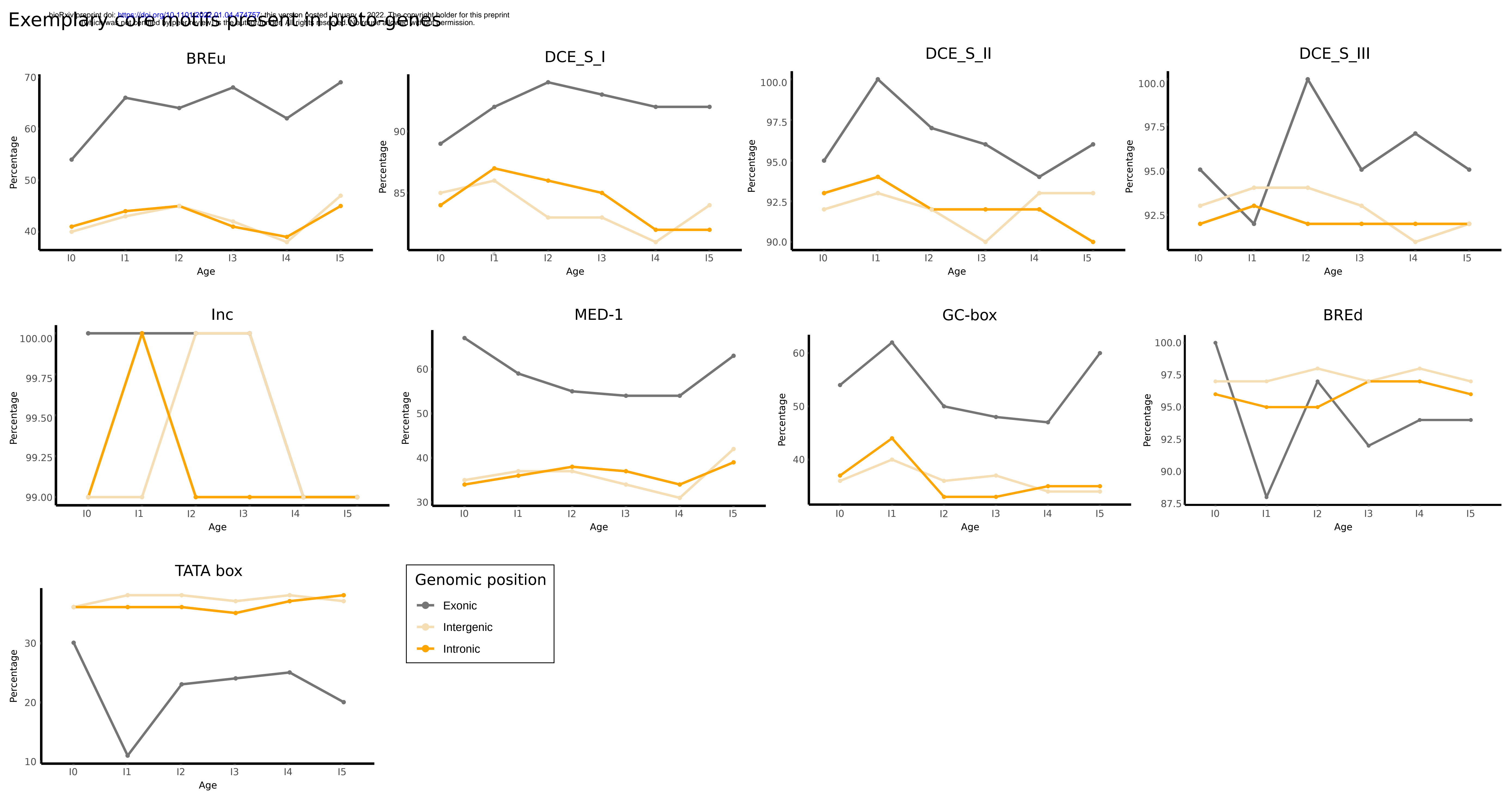


a) Energy distributions of 5 UTRs
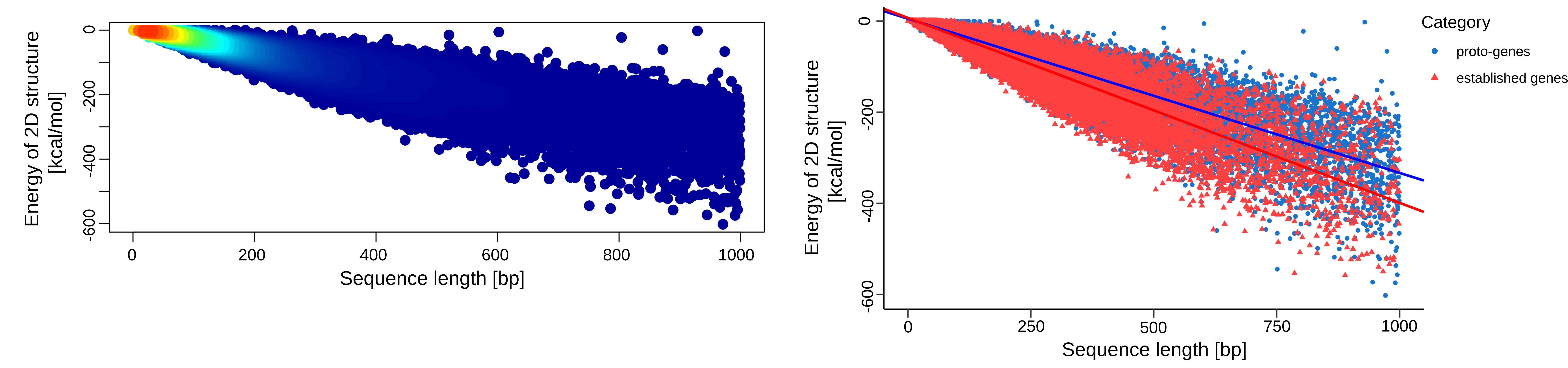

b) Structure probability

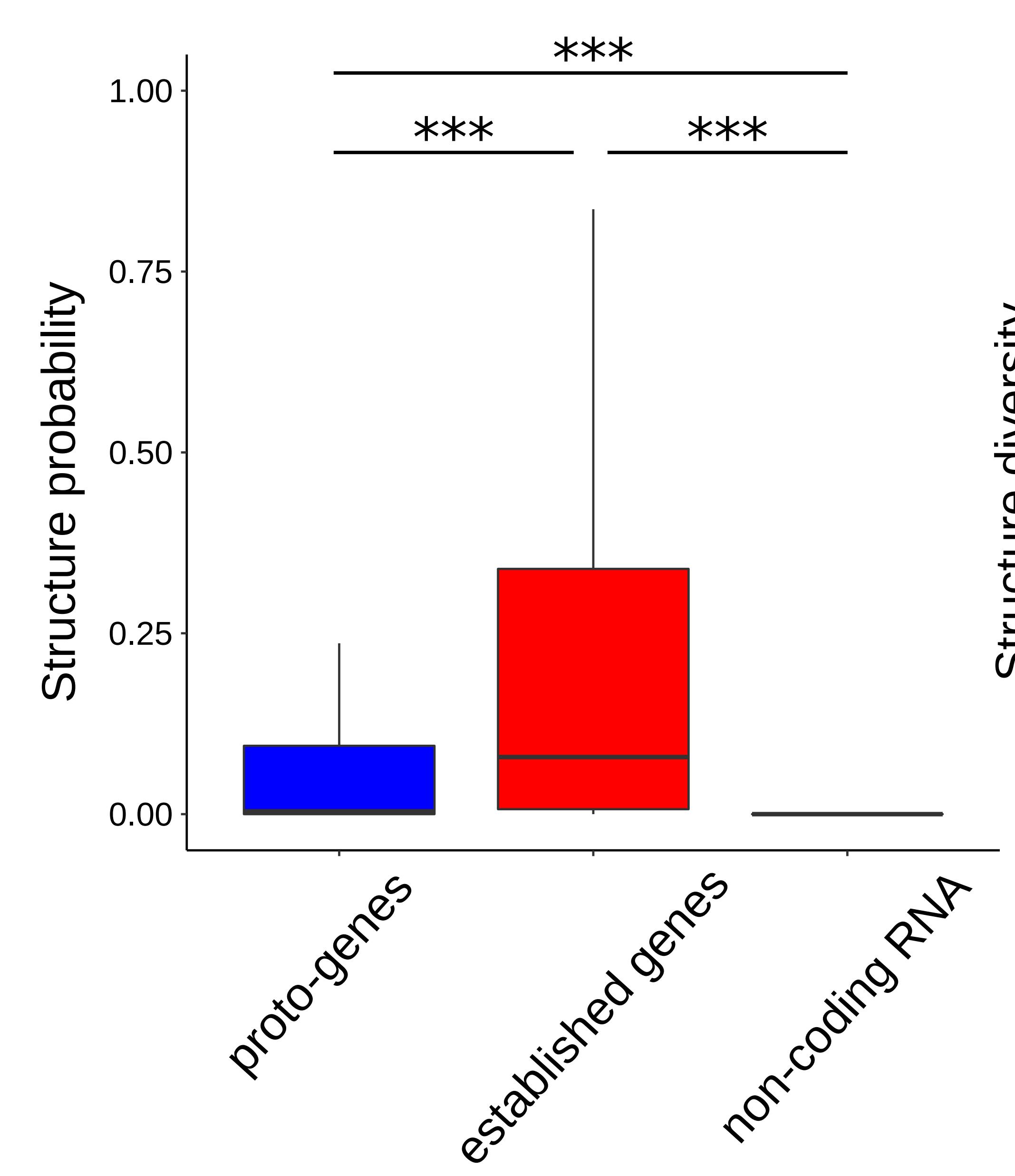

c) Structure diversity

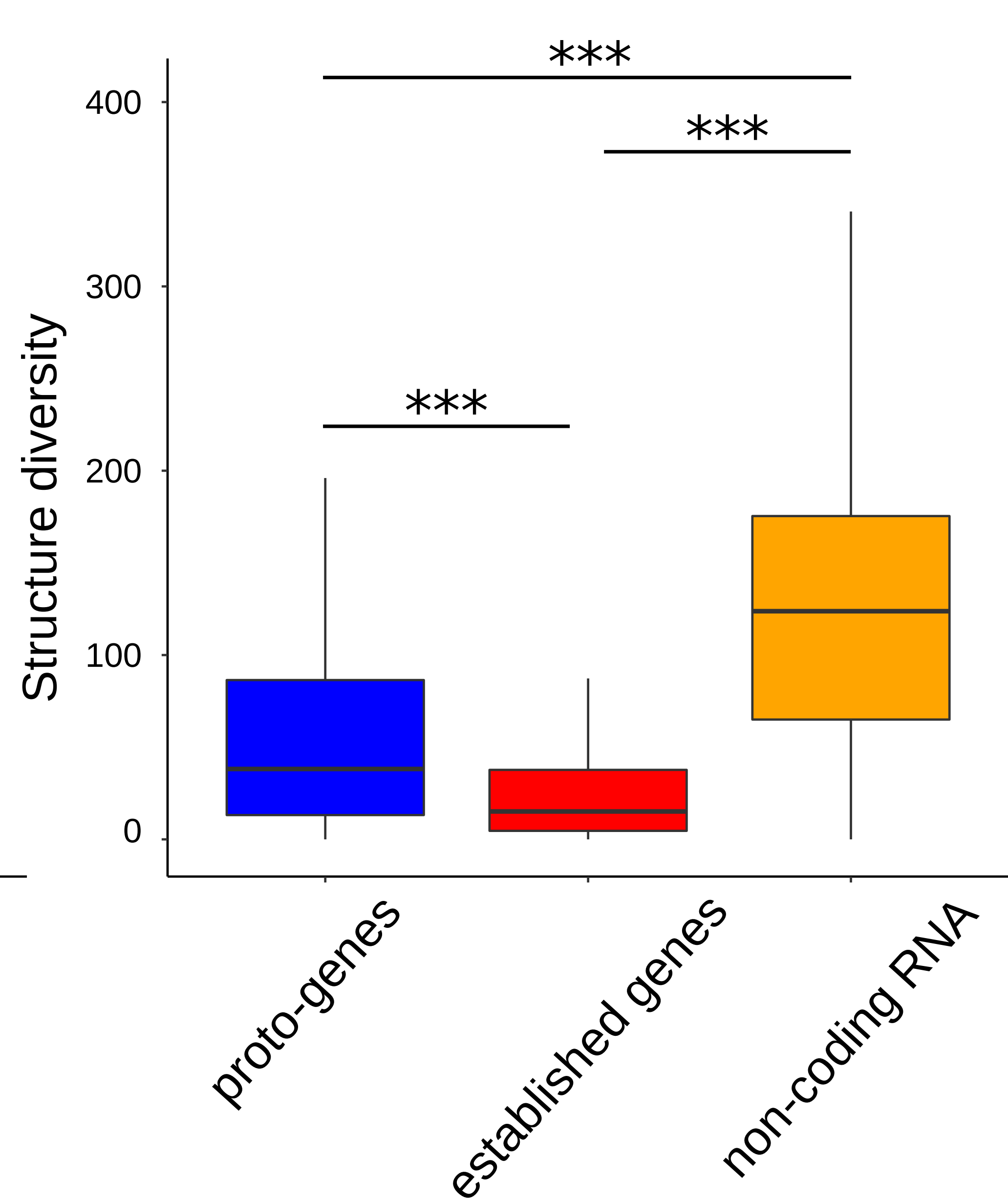

d) 2D structure of a representative 5'UTR region

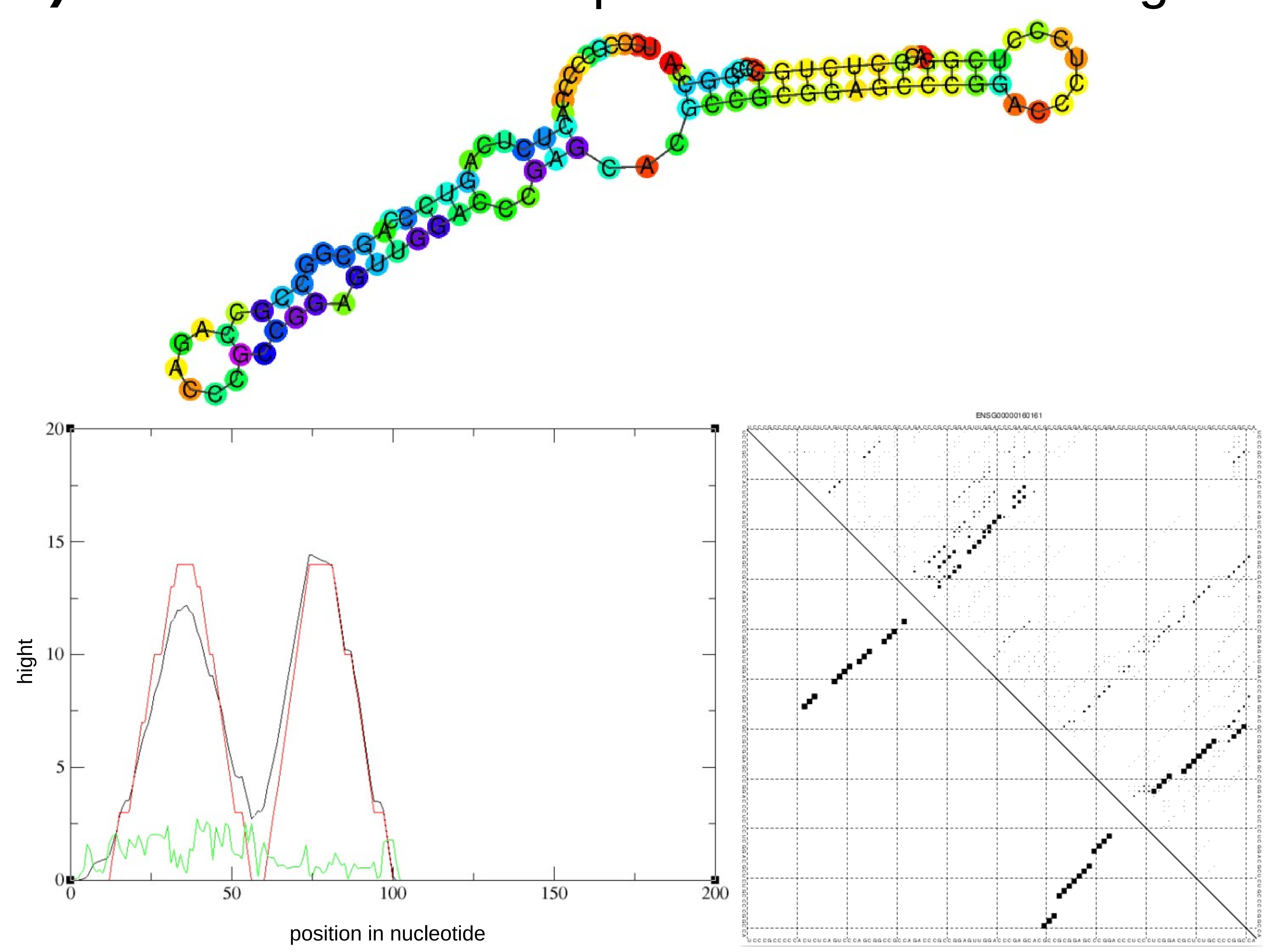


a) Percentage of proto-genes
with known domains

with known domains

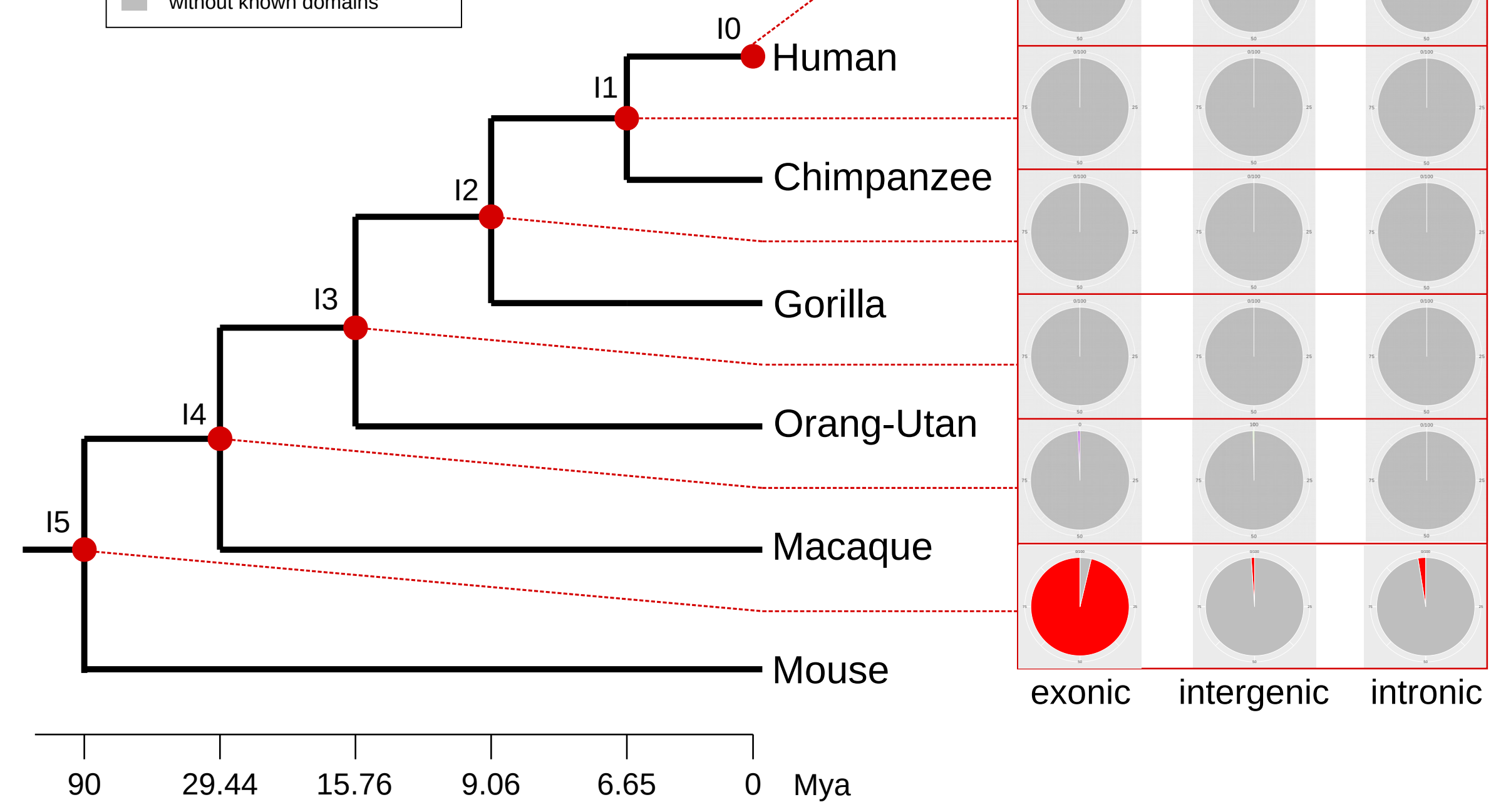

b)

Number of HCA domains according to age

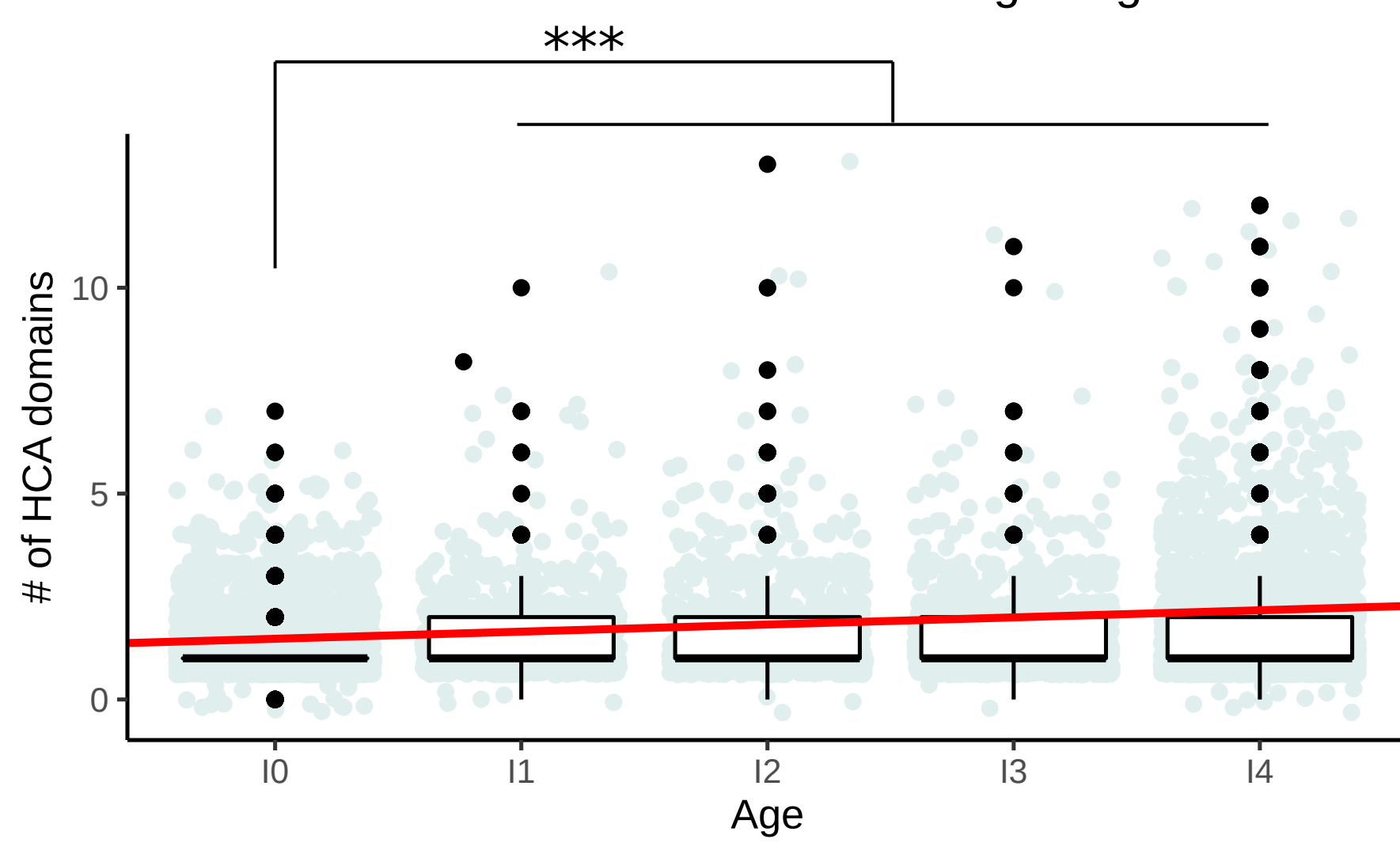

c) Number of HCA domains according to genomic position

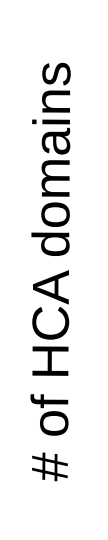

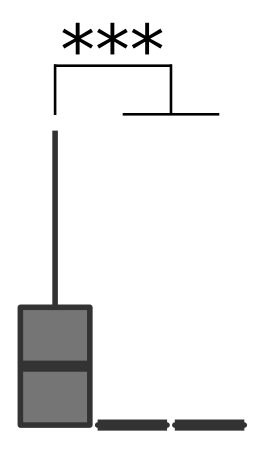

10

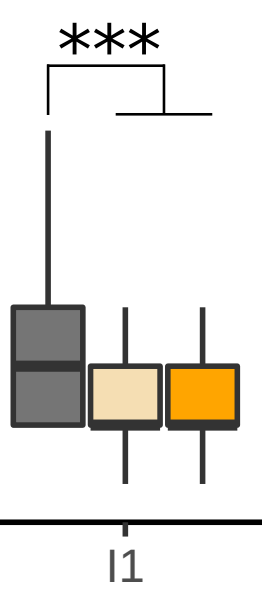

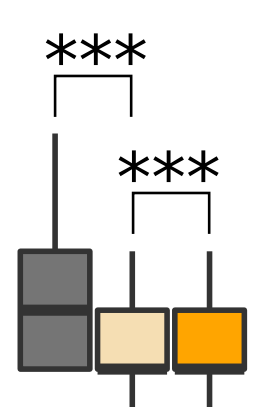

1

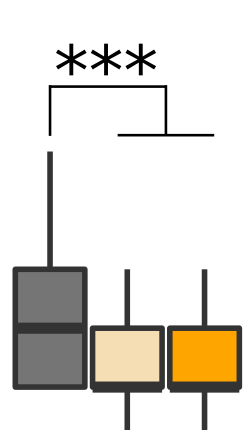

13

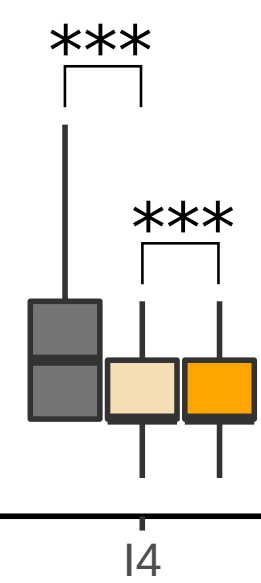

